\title{
Analysis of dengue model with fractal-fractional Caputo-Fabrizio operator
}

\author{
Fatmawati ${ }^{1}$, Muhammad Altaf Khan ${ }^{2,3^{*}} \mathbb{B}$, Cicik Alfiniyah' and Ebraheem Alzahrani ${ }^{4}$
}

${ }^{*}$ Correspondence:

muhammad.altaf.khan@tdtu.edu.vn

${ }^{2}$ Informetrics Research Group, Ton

Duc Thang University, Ho Chi Minh

City, Vietnam

${ }^{3}$ Faculty of Mathematics and

Statistics, Ton Duc Thang University,

Ho Chi Minh City, Vietnam

Full list of author information is

available at the end of the article

\section{是 Springer}

\begin{abstract}
In this work, we study the dengue dynamics with fractal-factional Caputo-Fabrizio operator. We employ real statistical data of dengue infection cases of East Java, Indonesia, from 2018 and parameterize the dengue model. The estimated basic reduction number for this dataset is $\mathcal{R}_{0} \approx 2.2020$. We briefly show the stability results of the model for the case when the basic reproduction number is $\mathcal{R}_{0}<1$. We apply the fractal-fractional operator in the framework of Caputo-Fabrizio to the model and present its numerical solution by using a novel approach. The parameter values estimated for the model are used to compare with fractal-fractional operator, and we suggest that the fractal-fractional operator provides the best fitting for real cases of dengue infection when varying the values of both operators' orders. We suggest some more graphical illustration for the model variables with various orders of fractal and fractional.
\end{abstract}

Keywords: Fractal-fractional; Caputo-Fabrizio operator; Dengue model; Real cases; Parameter estimates

Dengue infection is a vector-borne infection that is caused through a female mosquito from genus Flavivirus, family Flaviviridae. DENV1 to DENV4 are different serotypes associated with the dengue virus. A person infected with one DENV serotype has a lifetime of immunity from that serotype, but is not guaranteed immunity against other serotypes. Thus, it is possible for a person to be infected four times, once with each serotype [1]. Dengue fever is transmitted through the bite of female Aedes Aegypti and Aedes albopictus. Aedes Aegypti mosquitoes will attack healthy humans and people with dengue fever so that the virus enters the human body and reproduces itself; it will be in the blood for one week. If the human immune system is weak, it will be easy for humans to become dengue sufferers.

Dengue fever is a growing public health concern worldwide. As mobility and population density increase, the number of sufferers and the area of its spread are increasing. In recent years, dengue fever has become endemic in more than 100 countries including Africa, America, the Eastern Mediterranean, Southeast Asia, and the Western Pacific. The worst areas affected are in the United States, the Western Pacific, and Southeast Asia [2, 3]. These areas had the highest number of dengue cases, with a total of 1.2 million cases in 2008 and more than 3.34 million cases in 2016 reported [4].

(c) The Author(s) 2020 corrected publication 2021. This article is licensed under a Creative Commons Attribution 4.0 International License, which permits use, sharing, adaptation, distribution and reproduction in any medium or format, as long as you give appropriate credit to the original author(s) and the source, provide a link to the Creative Commons licence, and indicate if changes were made. The images or other third party material in this article are included in the article's Creative Commons licence, unless indicated otherwise in a credit line to the material. If material is not included in the article's Creative Commons licence and your intended use is not permitted by statutory regulation or exceeds the permitted use, you will need to obtain permission directly from the copyright holder. To view a copy of this licence, visit http://creativecommons.org/licenses/by/4.0/. 
In Indonesia, dengue was first discovered in the city of Surabaya in 1968, where as many as 58 people were infected and 24 of them died, with a mortality rate of $41.3 \%$. Since then, the disease has spread widely throughout Indonesia [5]. In 2015, there were 126,675 dengue fever sufferers across 34 provinces in Indonesia, and 1229 of them died. This number is higher than the previous year, as many as 907 patients died and there were 100,347 sufferers due to dengue fever. This increasing figure could be caused by climate change and low awareness to maintain environmental cleanliness [6].

Many researchers have utilized mathematical modeling to capture complexities of dengue infection transmission [7-14]. All of the models are stated in the form of ordinary integer-order derivative to describe the dynamics of the dengue infection. The limitation of these models is that they do not present the information about memory and learning mechanisms. In addition, it is difficult to explore the dynamics between two different points using the models based on integer-order derivatives. In order to address these concerns, various fractional-order derivative models were proposed in the literature (see $[15,16]$ and the references therein). Recently, some important results regarding the fractional operators were considered in [17-24]. In these works, the authors utilized the concepts of the fractional operators and obtained results for their proposed models.

Recently, some new results regarding the fractional operators in fractal-fractional the sense were considered in [16] and utilized in $[25,26]$ by the real statistical applications to banking data. The authors showed in their works that the fractal-fractional operators are effective for data fitting of real data. The fractal-fractional operators used in [25, 26] were in Caputo and Atangana-Baleanu sense. Due to the recent application of fractal-fractional operators, we aim in this work to apply the fractal-fractional operator in Caputo-Fabrizio sense and consider its application to real statistical data of Indonesia for dengue fever. Some more published work on the fractal-fractional and fractional modeling and its applications to science and engineering problems can be seen in [27-33]. For example, the authors considered a fractal-fractional model of an oscillator and presented the results in [27]. Avian influenza model with fractal-fractional derivative has been studied in [28]. The Valles model and its multiple attractors have been obtained using fractional derivative in [29]. A new chaotic model using fractal-fractional differentiation has been considered in [30]. A malaria model with fractal-fractional derivative was studied in [31]. Chaotic attractors with simple illustrations have been considered in [32]. The fractal-fractional applications to complex systems have been studied in [33].

The present work explores the dengue dynamics with hospitalization class in the framework of the fractal-fractional operator where the fractional operator is considered in the sense of Caputo-Fabrizio. Using this new idea, we formulate a dengue model and investigate its parameters using the real data of East Java, Indonesia, for the year 2018. We give a comparison of the fractal-fractional orders versus the real data of dengue cases and show some interesting results. We structure the rest of the paper as follows: The dengue model formulation and the related mathematical results are shown in Sect. 2. The basic concepts of fractal-fractional operators, the model construction using fractal-fractional operators, and associated results are shown in Sect. 3. The numerical solution of the fractal-fractional dengue model is presented in Sect. 4. Parameter estimates and the numerical results of the model using the fractal-fractional operator are discoursed in Sect. 5. Section 6 summarizes the work. 


\section{Dengue model transmission}

In this present section, we describe a host-vector model for dengue transmission. The host-vector model is divided into three mosquito populations, susceptible $\left(S_{m}\right)$, exposed $\left(E_{m}\right)$, and infectious $\left(I_{m}\right)$, and five human (host) populations, susceptible $\left(S_{h}\right)$, exposed $\left(E_{h}\right)$, infectious $\left(I_{h}\right)$, hospitalized and/or notified infectious $\left(P_{h}\right)$, and recovered $\left(R_{h}\right)$. Thus, the total human population, denoted by $N_{h}$, is given as $N_{h}=S_{h}+E_{h}+I_{h}+P_{h}+R_{h}$, where the total dynamics of mosquitos, denoted by $N_{m}$, is $N_{m}=S_{m}+E_{m}+I_{m}$. The system of differential equations that describes the host-vector model is written as

$$
\left\{\begin{array}{l}
\frac{d S_{m}}{d t}=\Pi_{m}-\tau \alpha_{h} S_{m} \frac{I_{h}}{N_{h}}-\mu_{m} S_{m}, \\
\frac{d E_{m}}{d t}=\tau \alpha_{h} S_{m} \frac{I_{h}}{N_{h}}-\left(\beta_{m}+\mu_{m}\right) E_{m}, \\
\frac{d I_{m}}{d t}=\beta_{m} E_{m}-\mu_{m} I_{m} \\
\frac{d S_{h}}{d t}=\Pi_{h}-\tau \alpha_{v} I_{m} \frac{S_{h}}{N_{h}}-v_{h} S_{h}, \\
\frac{d E_{h}}{d t}=\tau \alpha_{v} I_{m} \frac{S_{h}}{N_{h}}-\left(\beta_{h}+v_{h}\right) E_{h}, \\
\frac{d I_{h}}{d t}=\beta_{h} E_{h}-\left(\psi_{h}+\omega_{h}+v_{h}\right) I_{h} \\
\frac{d P_{h}}{d t}=\psi_{h} I_{h}-\left(\tau_{h}+\sigma_{h}+v_{h}\right) P_{h} \\
\frac{d R_{h}}{d t}=\omega_{h} I_{h}+\sigma_{h} P_{h}-v_{h} R_{h}
\end{array}\right.
$$

subject to the initial conditions

$$
\left\{\begin{array}{l}
S_{m}(0)=S_{m 0} \geq 0, \\
E_{m}(0)=E_{m 0} \geq 0, \\
I_{m}(0)=I_{m 0} \geq 0, \\
S_{h}(0)=S_{h 0} \geq 0, \\
E_{h}(0)=E_{h 0} \geq 0, \\
I_{h}(0)=I_{h 0} \geq 0, \\
P_{h}(0)=P_{h 0} \geq 0, \\
R_{h}(0)=R_{h 0} \geq 0 .
\end{array}\right.
$$

In model (1) above, the populations of vectors and humans are denoted by $\Pi_{m}$ and $\Pi_{h}$. The natural death rate of mosquitoes and humans are given by $\mu_{m}$ and $v_{h}$. The parameter $\tau$ measures the biting rate per mosquito per person. The transmission probability from an infected human to susceptible mosquitos, and the transmission probability from infected mosquitos to susceptible humans are shown respectively by $\alpha_{h}$ and $\alpha_{v}$. The intrinsic incubation period of mosquitos is given by $\beta_{m}$, and that of the humans is shown by $\beta_{h}$. The human infected cases that are notified and get hospitalization increase at a rate $\psi_{h}$. The infected human recover naturally at a rate $\omega_{h}$ while the hospitalized individuals get recovery at a rate $\sigma_{h}$. The hospitalized individuals die due to disease at a rate $\tau_{h}$. The complete details of the parameter descriptions of the dengue model (1) are presented briefly in Table 1.

\subsection{Positivity and boundedness of the solution}

In order to show that the dengue model given by (1) is epidemiologically meaningful, we have to prove that the associated state variables of the model stay nonnegative. It can also 
Table 1 Biological meaning of parameters for dengue model (1)

\begin{tabular}{ll}
\hline Parameter & Description \\
\hline$\Pi_{m}$ & Recruitment rate of mosquito \\
$\tau$ & Average biting rate per mosquito per person \\
$\alpha_{h}$ & Transmission probability from infected human to susceptible mosquito \\
$\mu_{m}$ & Natural death rate of mosquito \\
$\beta_{m}$ & Extrinsic incubation of mosquito \\
$\Pi_{h}$ & Recruitment rate of human \\
$\alpha_{v}$ & Transmission probability from infected mosquito to susceptible human \\
$\beta_{h}$ & Extrinsic incubation of human \\
$\psi_{h}$ & Rate of hospitalization and/or notification of infected human \\
$\omega_{h}$ & Natural recovery rate of infected human \\
$\sigma_{h}$ & Recovery rate of hospitalized and/or notified infected human \\
$\tau_{h}$ & Disease-related death rate of human \\
$\nu_{h}$ & Natural death rate of human \\
\hline
\end{tabular}

be explained that the solution of the dengue model with nonnegative initial conditions will remain nonnegative for every time greater than zero. We have the following lemma.

Lemma 1 Consider the initial data $G(0) \geq 0$, where

$$
G(t)=\left(S_{m}(t), E_{m}(t), I_{m}(t), S_{h}(t), E_{h}(t), I_{h}(t), P_{h}(t), R_{h}(t)\right) .
$$

Then the solutions of the model given by (1) are nonnegative for every time $t>0$. Further,

$$
\lim _{t \rightarrow \infty} N_{m}(t) \leq \frac{\Pi_{m}}{\mu_{m}} \text { and } \quad \lim _{t \rightarrow \infty} N_{h}(t) \leq \frac{\Pi_{h}}{v_{h}},
$$

with $N_{m}(t)=S_{m}(t)+E_{m}(t)+I_{m}(t)$ and $N_{h}(t)=S_{h}(t)+E_{h}(t)+I_{h}(t)+P_{h}(t)+R_{h}(t)$.

Proof Consider $t_{1}=\sup \{t>0: G(t)>0\}$. So, $t_{1}>0$. The first equation of the dengue model (1) leads to the following:

$$
\frac{d S_{m}}{d t}=\Pi_{m}-\tau \alpha_{h} S_{m} \frac{I_{h}}{N_{h}}-\mu_{m} S_{m}
$$

with $\lambda_{\nu}=\tau \alpha_{h} \frac{I_{h}}{N_{h}}$, then the above equation (3) becomes

$$
\frac{d S_{m}}{d t}=\Pi_{m}-\lambda_{v} S_{m}-\mu_{m} S_{m}
$$

The equation given by (4) can be expressed further as follows:

$$
\frac{d}{d t}\left\{S_{m}(t) \exp \left(\mu_{m} t+\int_{0}^{t} \lambda_{v}(\phi) d \phi\right)\right\}=\lambda_{\nu} \exp \left(\mu_{m} t+\int_{0}^{t} \lambda_{v}(\phi) d \phi\right) .
$$

Hence,

$$
\begin{gathered}
S_{m}\left(t_{1}\right) \exp \left(\mu_{m} t_{1}+\int_{0}^{t_{1}} \lambda_{v}(\phi) d \phi\right)-S_{m}(0) \\
=\lambda_{\nu} \exp \left(\mu_{m} y+\int_{0}^{y} \lambda_{v}(\psi) d \psi\right) d y,
\end{gathered}
$$


so that

$$
\begin{aligned}
S_{m}\left(t_{1}\right)= & S_{m}(0) \exp \left\{-\left(\mu_{m} t_{1}+\int_{0}^{t_{1}} \lambda_{\nu}(\phi) d \phi\right)\right\}+\exp \left\{-\left(\mu_{m} t_{1}+\int_{0}^{t_{1}} \lambda_{\nu}(\phi) d \phi\right)\right\} \\
& \times \int_{0}^{t_{1}} \lambda_{\nu} \exp \left(\mu_{m} y+\int_{0}^{y} \lambda_{\nu}(\psi) d \psi\right) d y>0 .
\end{aligned}
$$

Similar steps can be followed for the rest of the equations of the dengue model (1) that is, $G(t)>0$ for every $t>0$. In order to show the second part of the result, note that $0<$ $S_{m}(0) \leq N_{m}(t), 0<E_{m}(0) \leq N_{m}(t), 0<I_{m}(0) \leq N_{m}(t), 0<S_{h}(0) \leq N_{h}(t), 0 \leq E_{h}(0) \leq N_{h}(t)$, $0 \leq I_{h}(0) \leq N_{h}(t), 0 \leq P_{h}(0) \leq N_{h}(t), 0 \leq R_{h}(0) \leq N_{h}(t)$. Now, summing the mosquito and human compartments leads to the following:

$$
\frac{d N_{m}}{d t}=\Pi_{m}-\mu_{m} N_{v}
$$

and

$$
\frac{d N_{h}}{d t}=\Pi_{h}-v_{h} N_{h}
$$

Thus,

$$
\lim _{t \rightarrow \infty} N_{m}(t) \leq \frac{\Pi_{m}}{\mu_{m}} \quad \text { and } \quad \lim _{t \rightarrow \infty} N_{h}(t) \leq \frac{\Pi_{h}}{v_{h}}
$$

which is the required claim.

Next, we show the invariant regions for the given dengue model (1). Consider the feasible region $\Theta=\Theta_{v} \times \Theta_{h} \subset \mathbb{R}_{+}^{3} \times \mathbb{R}_{+}^{5}$, with

$$
\Theta_{v}=\left\{\left(S_{m}(t), E_{m}(t), I_{m}(t)\right) \in \mathbb{R}_{+}^{3}: N_{m}(t) \leq \frac{\Pi_{m}}{\mu_{m}}\right\}
$$

and

$$
\Theta_{h}=\left\{\left(S_{h}(t), E_{h}(t), I_{h}(t), P_{h}(t), R_{h}(t)\right) \in \mathbb{R}_{+}^{5}: N_{h}(t) \leq \frac{\Pi_{h}}{v_{h}}\right\} .
$$

We have the following results for this feasible region.

Lemma 2 The region given by $\Theta=\Theta_{v} \times \Theta_{h} \subset \mathbb{R}_{+}^{3} \times \mathbb{R}_{+}^{5}$ is positively invariant for the dengue model (1) with the nonnegative initial conditions in (2).

Proof The summation of the mosquito and human populations of the dengue model (1) leads to

$$
\frac{d N_{m}}{d t}=\Pi_{m}-\mu_{m} N_{m}
$$

and

$$
\frac{d N_{h}}{d t}=\Pi_{h}-v_{h} N_{h}
$$


Hence, $\frac{d N_{m}(t)}{d t} \leq 0$, if $N_{m}(0) \geq \frac{\Pi_{m}}{\mu_{m}}$ and $\frac{d N_{h}(t)}{d t} \leq 0$, if $N_{h}(0) \geq \frac{\Pi}{v_{h}}$. So, $N_{m}(t) \leq N_{m}(0) e^{-\mu_{m} t}+$ $\frac{\Pi_{m}}{\mu_{m}}\left(1-e^{-\mu_{m} t}\right)$ and $N_{h}(t) \leq N_{h}(0) e^{-v_{h} t}+\frac{\Pi_{h}}{v_{h}}\left(1-e^{-v_{h} t}\right)$. Thus, the region given by $\Theta$ is positively invariant. Also, if $N_{m}(0)>\frac{\Pi_{m}}{\mu_{m}}$ and $N_{h}(0)>\frac{\Pi_{h}}{v_{h}}$, then either the solutions enter $\Theta$ in finite time, or $N_{m}(t)$ tends to $\frac{\Pi_{m}}{\mu_{m}}$ and $N_{h}(t)$ tends to $\frac{\Pi_{h}}{v_{h}}$ asymptotically. So, the regions given by $\Theta$ attract all the solutions in $\mathbb{R}_{+}^{8}$.

\section{Preliminaries}

This section explores the related results regarding fractal-fractional calculus, the model construction in fractal-fractional Caputo-Fabrizio operator and model stability results. We consider first the basic of fractal-fractional (FF) calculus by following the literature in $[34,35]$.

Definition 1 If a function $g(t)$ is continuous and fractally differentiable over the given interval $\left(a_{1}, a_{2}\right)$ with order $\theta_{2}$, then the definition of the FF derivative of $g(t)$ with order $\theta_{1}$ in Riemann-Liouville sense with exponentially decaying kernel is given by:

$$
{ }^{F F E} D_{0, t}^{\theta_{1}, \theta_{2}}(g(t))=\frac{M\left(\theta_{1}\right)}{1-\theta_{1}} \frac{d}{d t^{\theta_{2}}} \int_{0}^{t} \exp \left(-\frac{\theta_{1}}{1-\theta_{1}}(t-s)\right) g(s) d s,
$$

with $\theta_{1}>0, \theta_{2} \leq m \in \mathbb{N}$ and $M(0)=M(1)=1$.

Definition 2 For a function $g(t)$ which is continuous and fractally differentiable over the given interval $\left(a_{1}, a_{2}\right)$, the definition of FF integral of $g(t)$ with order $\theta_{1}$ and exponentially decaying kernel is given by:

$$
{ }^{F F E} J_{0, t}^{\theta_{1}}(g(t))=\frac{\theta_{1} \theta_{2}}{M\left(\theta_{1}\right)} \int_{0}^{t} s^{\theta_{1}-1} g(s) d s+\frac{\theta_{2}\left(1-\theta_{1}\right) t^{\theta_{2}-1} g(t)}{M\left(\theta_{1}\right)} .
$$

\subsection{Fractal-fractional dengue model}

In the present subsection, we apply the fractal-fractional operator in the sense of CaputoFabrizio to the dengue model (1) described above. And we have

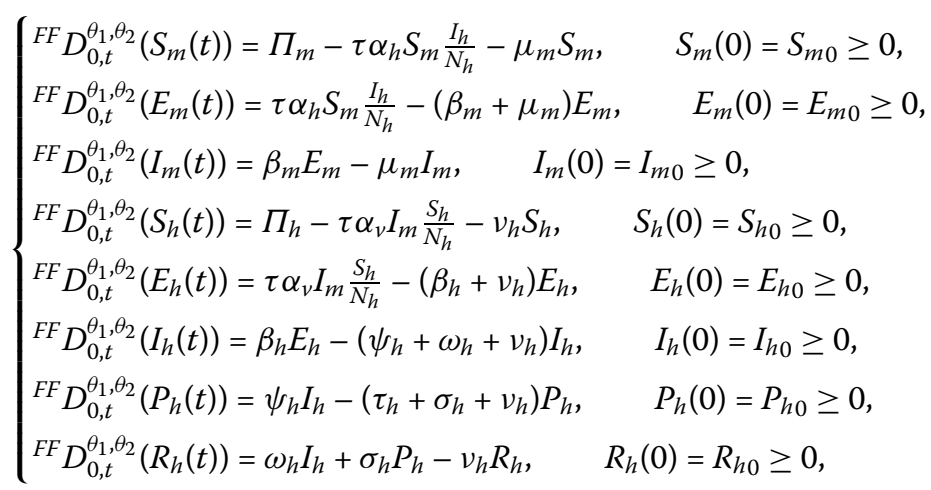

where $\theta_{1}$ and $\theta_{2}$ respectively represent the fractional and fractal order. The model (10) described above for the dengue infection using the fractal-fractional Caputo-Fabrizio operator is used further to obtain its numerical solution by using a novel numerical procedure in the following section. 


\subsection{Stability analysis of the disease-free case}

This section explores the stability results for the dengue model given at the disease-free equilibrium (DFE) at $E_{0}$. We set the right-hand side of the dengue model (10) equal to zero and obtain the following expressions:

$$
E_{0}=\left(S_{m}^{0}, 0,0, S_{h}^{0}, 0,0,0,0\right)=\left(\frac{\Pi_{m}}{\mu_{m}}, 0,0, \frac{\Pi_{h}}{v_{h}}, 0,0,0,0\right) .
$$

The stability of DFE at $E_{0}$ can be analyzed by using the next generation matrix of the dengue model (10). Considering the infected compartments in the dengue model (10), namely $E_{m}, I_{m}, E_{h}, I_{h}, P_{h}$, and following the instructions given in [36], the matrices $F$ and $V$ are obtained as follows:

$$
F=\left(\begin{array}{ccccc}
0 & 0 & 0 & \frac{\tau \alpha_{h} \nu_{h} \Pi_{m}}{\Pi_{h} \mu_{m}} & 0 \\
0 & 0 & 0 & 0 & 0 \\
0 & \tau \alpha_{v} & 0 & 0 & 0 \\
0 & 0 & 0 & 0 & 0 \\
0 & 0 & 0 & 0 & 0
\end{array}\right), \quad \text { and } \quad V=\left(\begin{array}{ccccc}
\pi_{1} & 0 & 0 & 0 & 0 \\
-\beta_{m} & \mu_{m} & 0 & 0 & 0 \\
0 & 0 & \pi_{2} & 0 & 0 \\
0 & 0 & -\beta_{h} & \pi_{3} & 0 \\
0 & 0 & 0 & -\psi_{h} & \pi_{4}
\end{array}\right) .
$$

where $\pi_{1}=\left(\beta_{m}+\mu_{m}\right), \pi_{2}=\left(\beta_{h}+v_{h}\right), \pi_{3}=\left(\psi_{h}+\omega_{h}+v_{h}\right)$, and $\pi_{4}=\left(\tau_{h}+\sigma_{h}+v_{h}\right)$. The required basic reproduction number of the given model is obtained through the spectral radius of the matrix $\mathcal{R}_{0}=\rho\left(F V^{-1}\right)$, which is given by the following expression:

$$
\mathcal{R}_{0}=\sqrt{\frac{\tau^{2} \alpha_{h} \beta_{h} \mu_{h} \alpha_{\nu} \beta_{m} \Pi_{m}}{\pi_{1} \pi_{2} \pi_{3} \Pi_{h} \mu_{m}^{2}}} .
$$

In the following theorem, we show that the dengue model given by (10) is locally asymptotically stable at $E_{0}$. We give the following result:

Theorem 1 The dengue model given by (10) at $E_{0}$ is locally asymptotically stable whenever $\mathcal{R}_{0}<1$.

Proof In order to prove the given theorem, we need to obtain the Jacobian matrix by evaluating the model (10) at $E_{0}$, and we have

$$
J\left(E_{0}\right)=\left(\begin{array}{cccccccc}
-\mu_{m} & 0 & 0 & 0 & 0 & -\frac{\tau \alpha_{h} \Pi_{v} v_{h}}{\Pi_{h} \mu_{m}} & 0 & 0 \\
0 & -\pi_{1} & 0 & 0 & 0 & \frac{\tau \alpha_{h} \Pi_{v} v_{h}}{\Pi_{h} \mu_{m}} & 0 & 0 \\
0 & \beta_{v} & -\mu_{m} & 0 & 0 & 0 & 0 & 0 \\
0 & 0 & -\tau \alpha_{v} & -v_{h} & 0 & 0 & 0 & 0 \\
0 & 0 & \tau \alpha_{v} & 0 & -\pi_{2} & 0 & 0 & 0 \\
0 & 0 & 0 & 0 & \gamma_{h} & -\pi_{3} & 0 & 0 \\
0 & 0 & 0 & 0 & 0 & \psi_{h} & -\pi_{4} & 0 \\
0 & 0 & 0 & 0 & 0 & \omega_{h} & \sigma_{h} & -v_{h}
\end{array}\right) .
$$

It can be seen from the above matrix $J\left(E_{0}\right)$ that the eigenvalues $-\mu_{m},-v_{h},-v_{h}$, and $-\pi_{4}$ are obviously negative, while the remaining four eigenvalues with negative real parts can be obtained through the following equation:

$$
\lambda^{4}+\varpi_{1} \lambda^{3}+\varpi_{2} \lambda^{2}+\varpi_{3} \lambda+\varpi_{4}=0
$$


where

$$
\begin{aligned}
& \varpi_{1}=\pi_{1}+\pi_{2}+\pi_{3}+\mu_{m}, \\
& \varpi_{2}=\pi_{3} \mu_{m}+\pi_{2}\left(\pi_{3}+\mu_{m}\right)+\pi_{1}\left(\pi_{2}+\pi_{3}+\mu_{m}\right), \\
& \varpi_{3}=\pi_{2} \pi_{3} \mu_{m}+\pi_{1}\left(\pi_{3} \mu_{m}+\pi_{2}\left(\pi_{3}+\mu_{m}\right)\right), \\
& \varpi_{4}=\pi_{1} \pi_{2} \pi_{3} \mu_{m}\left(1-\mathcal{R}_{0}^{2}\right) .
\end{aligned}
$$

The coefficients given by $\varpi_{i}$ for $i=1,2, \ldots, 4$ are obviously positive for $\varpi_{i}, i=1,2,3$ while $\varpi_{4}$ can be positive or negative based on the value of $\mathcal{R}_{0}$. For the DFE case, the value of the basic reproduction number should be less than 1 , so the last coefficient is positive when $\mathcal{R}_{0}<1$. So, for all the coefficients $\varpi_{i}$ for $i=1,2, \ldots, 4$ to be positive, they should satisfy the Rough-Hurtwiz criterion, which is easy to be satisfied, for the conditions supplied, $\varpi_{1} \varpi_{2} \varpi_{3}>\varpi_{3}^{2}+\varpi_{1}^{2} \varpi_{4}$, where $\varpi_{i}>0$ for all $i=1,2, \ldots, 4$. Thus, the condition of RoughHurtwiz criterion ensures the local asymptotic stability of the dengue model given by (10) at $E_{0}$.

\subsection{Endemic equilibria and their stability}

This subsection presents the endemic equilibria of the dengue model (10) denoted by $E_{1}=$ $\left(S_{m}^{*}, E_{m}^{*}, I_{m}^{*}, S_{h}^{*}, E_{h}^{*}, I_{h}^{*}, P_{h}^{*}, R_{h}^{*}\right)$ and given by

$$
\left\{\begin{array}{l}
S_{m}^{*}=\frac{\Pi_{m}}{\lambda_{v}^{*}+\mu_{m}}, \\
E_{m}^{*}=\frac{\lambda_{v}^{*} \Pi_{v}}{\pi_{1}\left(\lambda_{v}+\mu_{m}\right)}, \\
I_{m}^{*}=\frac{\beta_{m} \lambda_{v}^{*} \Pi_{m}}{\pi_{1} \mu_{m}\left(\lambda_{v}^{*}+\mu_{m}\right)}, \\
S_{h}^{*}=\frac{\Pi_{h}}{\lambda_{h}^{*}+v_{h}}, \\
E_{h}^{*}=\frac{\Pi_{h} \lambda_{h}^{*}}{\pi_{2}\left(\lambda_{h}^{*}+v_{h}\right)}, \\
I_{h}^{*}=\frac{\Pi_{h} \beta_{h} \lambda_{h}^{*}}{\pi_{2} \pi_{3}\left(\lambda_{h}^{*}+v_{h}\right)}, \\
P_{h}^{*}=\frac{\psi_{h} \Pi_{h} \beta_{h} \lambda_{h}^{*}}{\pi_{2} \pi_{3} \pi_{4}\left(\lambda_{h}^{*}+v_{h}\right)}, \\
R_{h}^{*}=\frac{\Pi_{h} \beta_{h} \lambda_{h}^{*}\left(\pi_{4} \omega_{h}+\psi_{h} \sigma_{h}\right)}{\pi_{2} \pi_{3} \pi_{4} v_{h}\left(\lambda_{h}^{*}+v_{h}\right)},
\end{array}\right.
$$

where

$$
\lambda_{v}^{*}=\frac{\tau \alpha_{h} I_{h}^{*}}{N_{h}^{*}}, \quad \lambda_{h}^{*}=\frac{\tau I_{m}^{*} \alpha_{v}}{N_{h}^{*}} .
$$

Using the expressions given by (11) in (12), this leads to the following:

$$
g_{1} \lambda_{h}^{* 2}+g_{2} \lambda_{h}^{*}+g_{3}=0
$$

where

$$
\begin{aligned}
g_{1}= & \pi_{1} \Pi_{h} \mu_{m}\left(\pi_{4}\left(v_{h}\left(\beta_{h}+\pi_{3}\right)+\omega_{h} \beta_{h}\right)+\psi_{h} \beta_{h}\left(v_{h}+\sigma_{h}\right)\right) \\
& \times\left(\pi_{4}\left(\tau \alpha_{h} \beta_{h} v_{h}+\mu_{m}\left(v_{h}\left(\beta_{h}+\pi_{3}\right)+\omega_{h} \beta_{h}\right)\right)+\psi_{h} \beta_{h} \mu_{m}\left(v_{h}+\sigma_{h}\right)\right), \\
g_{2}= & \pi_{2} \pi_{3} \pi_{4} v_{h}\left(\pi_{4}\left(2 \pi_{1} \Pi_{h} \mu_{m}^{2}\left(v_{h}\left(\beta_{h}+\pi_{3}\right)+\omega_{h} \beta_{h}\right)+\tau \alpha_{h} \beta_{h} v_{h}\left(\pi_{1} \Pi_{h} \mu_{m}-\tau \alpha_{v} \beta_{m} \Pi_{m}\right)\right)\right)
\end{aligned}
$$




$$
\begin{aligned}
& +2 \pi_{2} \pi_{3} \pi_{4} v_{h} \psi_{h} \pi_{1} \Pi_{h} \beta_{h} \mu_{m}^{2}\left(v_{h}+\sigma_{h}\right), \\
g_{3}= & \pi_{1} \pi_{2}^{2} \pi_{3}^{2} \pi_{4}^{2} \Pi_{h} v_{h}^{2} \mu_{m}^{2}\left(1-\mathcal{R}_{0}^{2}\right) .
\end{aligned}
$$

Here, $g_{1}>0, g_{3}$ depends on the sign of $\mathcal{R}_{0}$, and is positive when $\mathcal{R}_{0}<1$ and negative when $\mathcal{R}_{0}>1$. We establish the following result:

Theorem 2 The dengue model given by (10) has the following properties:

(i) If $g_{3}<0$ and $\mathcal{R}_{0}>1$, then there exists a unique endemic equilibrium;

(ii) If $g_{2}<0$ and $g_{3}=0$, then we have a unique endemic equilibrium;

(iii) If $g_{3}>0, g_{2}<0$, and their discriminant is positive, then two endemic equilibria exist; and

(iv) there are no equilibria otherwise.

It can be seen from the first point (i) of Theorem 2 that for the case of $\mathcal{R}_{0}>1$, we clearly have a unique positive endemic equilibrium. Theorem 2(iii) shows the possible existence of the backward bifurcation when $\mathcal{R}_{0}<1$.

\section{Novel solution procedure for fractal-fractional model}

For the numerical solution of the fractal-fractional dengue model (10), we present a novel procedure here that is based on the Adams-Bashforth technique. In order to have numerical scheme for the fractal-fractional model (10), we first express the model (10) in the following form:

$$
\left\{\begin{array}{l}
{ }^{C F} D_{0, t}^{\theta_{1}}\left(S_{m}(t)\right)=\theta_{2} t^{\theta_{2}-1} f_{1}\left(S_{m}, E_{m}, I_{m}, S_{h}, E_{h}, I_{h}, P_{h}, R_{h}, t\right), \\
{ }^{C F} D_{0, t}^{\theta_{1}}\left(E_{m}(t)\right)=\theta_{2} t^{\theta_{2}-1} f_{2}\left(S_{m}, E_{m}, I_{m}, S_{h}, E_{h}, I_{h}, P_{h}, R_{h}, t\right), \\
{ }^{C F} D_{0, t}^{\theta_{1}}\left(I_{m}(t)\right)=\theta_{2} t^{\theta_{2}-1} f_{3}\left(S_{m}, E_{m}, I_{m}, S_{h}, E_{h}, I_{h}, P_{h}, R_{h}, t\right), \\
{ }^{C F} D_{0, t}^{\theta_{1}}\left(S_{h}(t)\right)=\theta_{2} t^{\theta_{2}-1} f_{4}\left(S_{m}, E_{m}, I_{m}, S_{h}, E_{h}, I_{h}, P_{h}, R_{h}, t\right), \\
{ }^{C F} D_{0, t}^{\theta_{1}}\left(E_{h}(t)\right)=\theta_{2} t^{\theta_{2}-1} f_{5}\left(S_{m}, E_{m}, I_{m}, S_{h}, E_{h}, I_{h}, P_{h}, R_{h}, t\right), \\
{ }^{C F} D_{0, t}^{\theta_{1}}\left(I_{h}(t)\right)=\theta_{2} t^{\theta_{2}-1} f_{6}\left(S_{m}, E_{m}, I_{m}, S_{h}, E_{h}, I_{h}, P_{h}, R_{h}, t\right), \\
{ }^{C F} D_{0, t}^{\theta_{1}}\left(R_{h}(t)\right)=\theta_{2} t^{\theta_{2}-1} f_{7}\left(S_{m}, E_{m}, I_{m}, S_{h}, E_{h}, I_{h}, P_{h}, R_{h}, t\right), \\
{ }^{C F} D_{0, t}^{\theta_{1}}\left(I_{m}(t)\right)=\theta_{2} t^{\theta_{2}-1} f_{8}\left(S_{m}, E_{m}, I_{m}, S_{h}, E_{h}, I_{h}, P_{h}, R_{h}, t\right) .
\end{array}\right.
$$

We apply the CF integral on equation (13), which leads to the following:

$$
\begin{aligned}
& S_{m}(t)=S_{m}(0)+\frac{\theta_{2}}{\Gamma\left(\theta_{1}\right)} \int_{0}^{t} \lambda^{\theta_{2}-1}(t-\lambda)^{\theta_{1}-1} f_{1}\left(S_{m}, E_{m}, I_{m}, S_{h}, E_{h}, I_{h}, P_{h}, R_{h}, \lambda\right) d \lambda, \\
& E_{m}(t)=E_{m}(0)+\frac{\theta_{2}}{\Gamma\left(\theta_{1}\right)} \int_{0}^{t} \lambda^{\theta_{2}-1}(t-\lambda)^{\theta_{1}-1} f_{2}\left(S_{m}, E_{m}, I_{m}, S_{h}, E_{h}, I_{h}, P_{h}, R_{h}, \lambda\right) d \lambda, \\
& I_{m}(t)=I_{m}(0)+\frac{\theta_{2}}{\Gamma\left(\theta_{1}\right)} \int_{0}^{t} \lambda^{\theta_{2}-1}(t-\lambda)^{\theta_{1}-1} f_{3}\left(S_{m}, E_{m}, I_{m}, S_{h}, E_{h}, I_{h}, P_{h}, R_{h}, \lambda\right) d \lambda, \\
& S_{h}(t)=S_{h}(0)+\frac{\theta_{2}}{\Gamma\left(\theta_{1}\right)} \int_{0}^{t} \lambda^{\theta_{2}-1}(t-\lambda)^{\theta_{1}-1} f_{4}\left(S_{m}, E_{m}, I_{m}, S_{h}, E_{h}, I_{h}, P_{h}, R_{h}, \lambda\right) d \lambda, \\
& E_{h}(t)=E_{h}(0)+\frac{\theta_{2}}{\Gamma\left(\theta_{1}\right)} \int_{0}^{t} \lambda^{\theta_{2}-1}(t-\lambda)^{\theta_{1}-1} f_{5}\left(S_{m}, E_{m}, I_{m}, S_{h}, E_{h}, I_{h}, P_{h}, R_{h}, \lambda\right) d \lambda,
\end{aligned}
$$




$$
\begin{aligned}
I_{h}(t)= & I_{h}(0)+\frac{\theta_{2}}{\Gamma\left(\theta_{1}\right)} \int_{0}^{t} \lambda^{\theta_{2}-1}(t-\lambda)^{\theta_{1}-1} f_{6}\left(S_{m}, E_{m}, I_{m}, S_{h}, E_{h}, I_{h}, P_{h}, R_{h}, \lambda\right) d \lambda \\
P_{h}(t)= & P_{h}(0)+\frac{\theta_{2}}{\Gamma\left(\theta_{1}\right)} \int_{0}^{t} \lambda^{\theta_{2}-1}(t-\lambda)^{\theta_{1}-1} f_{7}\left(S_{m}, E_{m}, I_{m}, S_{h}, E_{h}, I_{h}, P_{h}, R_{h}, \lambda\right) d \lambda \\
R_{h}(t)= & R_{h}(0)+\frac{\theta_{2}}{\Gamma\left(\theta_{1}\right)} \int_{0}^{t} \lambda^{\theta_{2}-1}(t-\lambda)^{\theta_{1}-1} \\
& \times f_{8}\left(S_{m}, E_{m}, I_{m}, S_{h}, E_{h}, I_{h}, P_{h}, R_{h}, \lambda\right) d \lambda
\end{aligned}
$$

where

$$
\begin{aligned}
& f_{1}\left(S_{m}, E_{m}, I_{m}, S_{h}, E_{h}, I_{h}, P_{h}, R_{h}, \lambda\right)=\Pi_{m}-\tau \alpha_{h} S_{m} \frac{I_{h}}{N_{h}}-\mu_{m} S_{m}, \\
& f_{2}\left(S_{m}, E_{m}, I_{m}, S_{h}, E_{h}, I_{h}, P_{h}, R_{h}, \lambda\right)=\tau \alpha_{h} S_{m} \frac{I_{h}}{N_{h}}-\left(\beta_{m}+\mu_{m}\right) E_{m}, \\
& f_{3}\left(S_{m}, E_{m}, I_{m}, S_{h}, E_{h}, I_{h}, P_{h}, R_{h}, \lambda\right)=\beta_{m} E_{m}-\mu_{m} I_{m}, \\
& f_{4}\left(S_{m}, E_{m}, I_{m}, S_{h}, E_{h}, I_{h}, P_{h}, R_{h}, \lambda\right)=\Pi_{h}-\tau \alpha_{v} I_{m} \frac{S_{h}}{N_{h}}-v_{h} S_{h}, \\
& f_{5}\left(S_{m}, E_{m}, I_{m}, S_{h}, E_{h}, I_{h}, P_{h}, R_{h}, \lambda\right)=\tau \alpha_{v} I_{m} \frac{S_{h}}{N_{h}}-\left(\beta_{h}+v_{h}\right) E_{h}, \\
& f_{6}\left(S_{m}, E_{m}, I_{m}, S_{h}, E_{h}, I_{h}, P_{h}, R_{h}, \lambda\right)=\beta_{h} E_{h}-\left(\psi_{h}+\omega_{h}+v_{h}\right) I_{h}, \\
& f_{7}\left(S_{m}, E_{m}, I_{m}, S_{h}, E_{h}, I_{h}, P_{h}, R_{h}, \lambda\right)=\psi_{h} I_{h}-\left(\tau_{h}+\sigma_{h}+v_{h}\right) P_{h}, \\
& f_{8}\left(S_{m}, E_{m}, I_{m}, S_{h}, E_{h}, I_{h}, P_{h}, R_{h}, \lambda\right)=\omega_{h} I_{h}+\sigma_{h} P_{h}-v_{h} R_{h} .
\end{aligned}
$$

We are presenting now a novel approach for the above model and use the approach at $t_{n+1}$. We have the following:

$$
\begin{aligned}
S_{m}(t)= & S_{m}^{0}+\frac{\theta_{2} t^{\theta_{2}-1}\left(1-\theta_{1}\right)}{M\left(\theta_{1}\right)} f_{1}\left(S_{m}, E_{m}, I_{m}, S_{h}, E_{h}, I_{h}, P_{h}, R_{h}, t\right) \\
& +\frac{\theta_{1} \theta_{2}}{M\left(\theta_{1}\right)} \int_{0}^{t} \lambda^{\theta_{2}-1} f_{1}\left(S_{m}, E_{m}, I_{m}, S_{h}, E_{h}, I_{h}, P_{h}, R_{h}, \lambda\right) d \lambda, \\
E_{m}(t)= & E_{m}^{0}+\frac{\theta_{2} t^{\theta_{2}-1}\left(1-\theta_{1}\right)}{M\left(\theta_{1}\right)} f_{2}\left(S_{m}, E_{m}, I_{m}, S_{h}, E_{h}, I_{h}, P_{h}, R_{h}, t\right) \\
& +\frac{\theta_{1} \theta_{2}}{M\left(\theta_{1}\right)} \int_{0}^{t} \lambda^{\theta_{2}-1} f_{2}\left(S_{m}, E_{m}, I_{m}, S_{h}, E_{h}, I_{h}, P_{h}, R_{h}, \lambda\right) d \lambda, \\
I_{m}(t)= & I_{m}^{0}+\frac{\theta_{2} t^{\theta_{2}-1}\left(1-\theta_{1}\right)}{M\left(\theta_{1}\right)} f_{3}\left(S_{m}, E_{m}, I_{m}, S_{h}, E_{h}, I_{h}, P_{h}, R_{h}, t\right) \\
& +\frac{\theta_{1} \theta_{2}}{M\left(\theta_{1}\right)} \int_{0}^{t} \lambda^{\theta_{2}-1} f_{3}\left(S_{m}, E_{m}, I_{m}, S_{h}, E_{h}, I_{h}, P_{h}, R_{h}, \lambda\right) d \lambda, \\
S_{h}(t)= & S_{h}^{0}+\frac{\theta_{2} t^{\theta_{2}-1}\left(1-\theta_{1}\right)}{M\left(\theta_{1}\right)} f_{4}\left(S_{m}, E_{m}, I_{m}, S_{h}, E_{h}, I_{h}, P_{h}, R_{h}, t\right) \\
& +\frac{\theta_{1} \theta_{2}}{M\left(\theta_{1}\right)} \int_{0}^{t} \lambda^{\theta_{2}-1} f_{4}\left(S_{m}, E_{m}, I_{m}, S_{h}, E_{h}, I_{h}, P_{h}, R_{h}, \lambda\right) d \lambda, \\
E_{h}(t)= & E_{h}^{0}+\frac{\theta_{2} t^{\theta_{2}-1}\left(1-\theta_{1}\right)}{M\left(\theta_{1}\right)} f_{5}\left(S_{m}, E_{m}, I_{m}, S_{h}, E_{h}, I_{h}, P_{h}, R_{h}, t\right)
\end{aligned}
$$




$$
\begin{aligned}
& +\frac{\theta_{1} \theta_{2}}{M\left(\theta_{1}\right)} \int_{0}^{t} \lambda^{\theta_{2}-1} f_{5}\left(S_{m}, E_{m}, I_{m}, S_{h}, E_{h}, I_{h}, P_{h}, R_{h}, \lambda\right) d \lambda, \\
I_{h}(t)= & I_{h}^{0}+\frac{\theta_{2} t^{\theta_{2}-1}\left(1-\theta_{1}\right)}{M\left(\theta_{1}\right)} f_{6}\left(S_{m}, E_{m}, I_{m}, S_{h}, E_{h}, I_{h}, P_{h}, R_{h}, t\right) \\
& +\frac{\theta_{1} \theta_{2}}{M\left(\theta_{1}\right)} \int_{0}^{t} \lambda^{\theta_{2}-1} f_{6}\left(S_{m}, E_{m}, I_{m}, S_{h}, E_{h}, I_{h}, P_{h}, R_{h}, \lambda\right) d \lambda, \\
P_{h}(t)= & P_{h}^{0}+\frac{\theta_{2} \theta^{\theta_{2}-1}\left(1-\theta_{1}\right)}{M\left(\theta_{1}\right)} f_{7}\left(S_{m}, E_{m}, I_{m}, S_{h}, E_{h}, I_{h}, P_{h}, R_{h}, t\right) \\
& +\frac{\theta_{1} \theta_{2}}{M\left(\theta_{1}\right)} \int_{0}^{t} \lambda^{\theta_{2}-1} f_{7}\left(S_{m}, E_{m}, I_{m}, S_{h}, E_{h}, I_{h}, P_{h}, R_{h}, \lambda\right) d \lambda, \\
R_{h}(t)= & R_{h}^{0}+\frac{\theta_{2} t^{\theta_{2}-1}\left(1-\theta_{1}\right)}{M\left(\theta_{1}\right)} f_{8}\left(S_{m}, E_{m}, I_{m}, S_{h}, E_{h}, I_{h}, P_{h}, R_{h}, t\right) \\
& +\frac{\theta_{1} \theta_{2}}{M\left(\theta_{1}\right)} \int_{0}^{t} \lambda^{\theta_{2}-1} f_{8}\left(S_{m}, E_{m}, I_{m}, S_{h}, E_{h}, I_{h}, P_{h}, R_{h}, \lambda\right) d \lambda .
\end{aligned}
$$

At $t_{n+1}$, we have the following:

$$
\begin{aligned}
S_{m}^{n+1}= & S_{m}^{0}+\frac{\theta_{2} t^{\theta_{2}-1}\left(1-\theta_{1}\right)}{M\left(\theta_{1}\right)} f_{1}\left(S_{m}^{n}, E_{m}^{n}, I_{m}^{n}, S_{h}^{n}, E_{h}^{n}, I_{h}^{n}, P_{h}^{n}, R_{h}^{n}, t_{n}\right) \\
& +\frac{\theta_{1} \theta_{2}}{M\left(\theta_{1}\right)} \int_{0}^{t_{n+1}} \lambda^{\theta_{2}-1} f_{1}\left(S_{m}, E_{m}, I_{m}, S_{h}, E_{h}, I_{h}, P_{h}, R_{h}, \lambda\right) d \lambda, \\
E_{m}^{n+1}= & E_{m}^{0}+\frac{\theta_{2} t^{\theta_{2}-1}\left(1-\theta_{1}\right)}{M\left(\theta_{1}\right)} f_{2}\left(S_{m}^{n}, E_{m}^{n}, I_{m}^{n}, S_{h}^{n}, E_{h}^{n}, I_{h}^{n}, P_{h}^{n}, R_{h}^{n}, t_{n}\right) \\
& +\frac{\theta_{1} \theta_{2}}{M\left(\theta_{1}\right)} \int_{0}^{t_{n+1}} \lambda^{\theta_{2}-1} f_{2}\left(S_{m}, E_{m}, I_{m}, S_{h}, E_{h}, I_{h}, P_{h}, R_{h}, \lambda\right) d \lambda, \\
I_{m}^{n+1}= & I_{m}^{0}+\frac{\theta_{2} t^{\theta_{2}-1}\left(1-\theta_{1}\right)}{M\left(\theta_{1}\right)} f_{3}\left(S_{m}^{n}, E_{m}^{n}, I_{m}^{n}, S_{h}^{n}, E_{h}^{n}, I_{h}^{n}, P_{h}^{n}, R_{h}^{n}, t_{n}\right) \\
& +\frac{\theta_{1} \theta_{2}}{M\left(\theta_{1}\right)} \int_{0}^{t_{n+1}} \lambda^{\theta_{2}-1} f_{3}\left(S_{m}, E_{m}, I_{m}, S_{h}, E_{h}, I_{h}, P_{h}, R_{h}, \lambda\right) d \lambda, \\
S_{h}^{n+1}= & S_{h}^{0}+\frac{\theta_{2} t^{\theta_{2}-1}\left(1-\theta_{1}\right)}{M\left(\theta_{1}\right)} f_{4}\left(S_{m}^{n}, E_{m}^{n}, I_{m}^{n}, S_{h}^{n}, E_{h}^{n}, I_{h}^{n}, P_{h}^{n}, R_{h}^{n}, t_{n}\right) \\
& +\frac{\theta_{1} \theta_{2}}{M\left(\theta_{1}\right)} \int_{0}^{t_{n+1}} \lambda^{\theta_{2}-1} f_{4}\left(S_{m}, E_{m}, I_{m}, S_{h}, E_{h}, I_{h}, P_{h}, R_{h}, \lambda\right) d \lambda, \\
P_{h}^{n+1}= & P_{h}^{0}+\frac{\theta_{2} t^{\theta_{2}-1}\left(1-\theta_{1}\right)}{M\left(\theta_{1}\right)} f_{7}\left(S_{m}^{n}, E_{m}^{n}, I_{m}^{n}, S_{h}^{n}, E_{h}^{n}, I_{h}^{n}, P_{h}^{n}, R_{h}^{n}, t_{n}\right) \\
E_{h}^{n+1}= & E_{h}^{0}+\frac{\theta_{2} t^{\theta_{2}-1}\left(1-\theta_{1}\right)}{M\left(\theta_{1}\right)} f_{5}\left(S_{m}^{n}, E_{m}^{n}, I_{m}^{n}, S_{h}^{n}, E_{h}^{n}, I_{h}^{n}, P_{h}^{n}, R_{h}^{n}, t_{n}\right) \\
& +\frac{\theta_{1} \theta_{2}}{M\left(\theta_{1}\right)} \int_{0}^{t_{n+1}} \lambda^{\theta_{2}-1} f_{5}\left(S_{m}, E_{m}, I_{m}, S_{h}, E_{h}, I_{h}, P_{h}, R_{h}, \lambda\right) d \lambda, \\
I_{h}^{n+1}= & I_{h}^{0}+\frac{\theta_{2} t^{\theta_{2}-1}\left(1-\theta_{1}\right)}{M\left(\theta_{1}\right)} f_{6}\left(S_{m}^{n}, E_{m}^{n}, I_{m}^{n}, S_{h}^{n}, E_{h}^{n}, I_{h}^{n}, P_{h}^{n}, R_{h}^{n}, t_{n}\right) \\
& \frac{\theta_{1} \theta_{2}}{\left.t_{n+1} \theta_{1}\right)} \lambda^{\theta_{2}-1} f_{6}\left(S_{m}, E_{m}, I_{m}, S_{h}, E_{h}, I_{h}, P_{h}, R_{h}, \lambda\right) d \lambda, \\
&
\end{aligned}
$$




$$
\begin{aligned}
& +\frac{\theta_{1} \theta_{2}}{M\left(\theta_{1}\right)} \int_{0}^{t_{n+1}} \lambda^{\theta_{2}-1} f_{7}\left(S_{m}, E_{m}, I_{m}, S_{h}, E_{h}, I_{h}, P_{h}, R_{h}, \lambda\right) d \lambda, \\
R_{h}^{n+1}= & R_{h}^{0}+\frac{\theta_{2} t^{\theta_{2}-1}\left(1-\theta_{1}\right)}{M\left(\theta_{1}\right)} f_{8}\left(S_{m}^{n}, E_{m}^{n}, I_{m}^{n}, S_{h}^{n}, E_{h}^{n}, I_{h}^{n}, P_{h}^{n}, R_{h}^{n}, t_{n}\right) \\
& +\frac{\theta_{1} \theta_{2}}{M\left(\theta_{1}\right)} \int_{0}^{t_{n+1}} \lambda^{\theta_{2}-1} f_{8}\left(S_{m}, E_{m}, I_{m}, S_{h}, E_{h}, I_{h}, P_{h}, R_{h}, \lambda\right) d \lambda .
\end{aligned}
$$

The following is obtained by taking the difference between consecutive terms:

$$
\begin{aligned}
& S_{m}^{n+1}=S_{m}^{n}+\frac{\theta_{2} t^{\theta_{2}-1}\left(1-\theta_{1}\right)}{M\left(\theta_{1}\right)} f_{1}\left(S_{m}^{n}, E_{m}^{n}, I_{m}^{n}, S_{h}^{n}, E_{h}^{n}, I_{h}^{n}, P_{h}^{n}, R_{h}^{n}, t_{n}\right) \\
& -\frac{\theta_{2} t_{n-1}^{\theta_{2}-1}\left(1-\theta_{1}\right)}{M\left(\theta_{1}\right)} f_{1}\left(S_{m}^{n-1}, E_{m}^{n-1}, I_{m}^{n-1}, S_{h}^{n-1}, E_{h}^{n-1}, I_{h}^{n-1}, P_{h}^{n-1}, R_{h}^{n-1}, t_{n-1}\right) \\
& +\frac{\theta_{1} \theta_{2}}{M\left(\theta_{1}\right)} \int_{t_{n}}^{t_{n+1}} \lambda^{\theta_{2}-1} f_{1}\left(S_{m}, E_{m}, I_{m}, S_{h}, E_{h}, I_{h}, P_{h}, R_{h}, \lambda\right) d \lambda, \\
& E_{m}^{n+1}=E_{m}^{n}+\frac{\theta_{2} t^{\theta_{2}-1}\left(1-\theta_{1}\right)}{M\left(\theta_{1}\right)} f_{2}\left(S_{m}^{n}, E_{m}^{n}, I_{m}^{n}, S_{h}^{n}, E_{h}^{n}, I_{h}^{n}, P_{h}^{n}, R_{h}^{n}, t_{n}\right) \\
& -\frac{\theta_{2} t_{n-1}^{\theta_{2}-1}\left(1-\theta_{1}\right)}{M\left(\theta_{1}\right)} f_{2}\left(S_{m}^{n-1}, E_{m}^{n-1}, I_{m}^{n-1}, S_{h}^{n-1}, E_{h}^{n-1}, I_{h}^{n-1}, P_{h}^{n-1}, R_{h}^{n-1}, t_{n-1}\right) \\
& +\frac{\theta_{1} \theta_{2}}{M\left(\theta_{1}\right)} \int_{t_{n}}^{t_{n+1}} \lambda^{\theta_{2}-1} f_{2}\left(S_{m}, E_{m}, I_{m}, S_{h}, E_{h}, I_{h}, P_{h}, R_{h}, \lambda\right) d \lambda, \\
& I_{m}^{n+1}=I_{m}^{n}+\frac{\theta_{2} t^{\theta_{2}-1}\left(1-\theta_{1}\right)}{M\left(\theta_{1}\right)} f_{3}\left(S_{m}^{n}, E_{m}^{n}, I_{m}^{n}, S_{h}^{n}, E_{h}^{n}, I_{h}^{n}, P_{h}^{n}, R_{h}^{n}, t_{n}\right) \\
& -\frac{\theta_{2} t_{n-1}^{\theta_{2}-1}\left(1-\theta_{1}\right)}{M\left(\theta_{1}\right)} f_{3}\left(S_{m}^{n-1}, E_{m}^{n-1}, I_{m}^{n-1}, S_{h}^{n-1}, E_{h}^{n-1}, I_{h}^{n-1}, P_{h}^{n-1}, R_{h}^{n-1}, t_{n-1}\right) \\
& +\frac{\theta_{1} \theta_{2}}{M\left(\theta_{1}\right)} \int_{t_{n}}^{t_{n+1}} \lambda^{\theta_{2}-1} f_{3}\left(S_{m}, E_{m}, I_{m}, S_{h}, E_{h}, I_{h}, P_{h}, R_{h}, \lambda\right) d \lambda, \\
& S_{h}^{n+1}=S_{h}^{n}+\frac{\theta_{2} t^{\theta_{2}-1}\left(1-\theta_{1}\right)}{M\left(\theta_{1}\right)} f_{4}\left(S_{m}^{n}, E_{m}^{n}, I_{m}^{n}, S_{h}^{n}, E_{h}^{n}, I_{h}^{n}, P_{h}^{n}, R_{h}^{n}, t_{n}\right) \\
& -\frac{\theta_{2} t_{n-1}^{\theta_{2}-1}\left(1-\theta_{1}\right)}{M\left(\theta_{1}\right)} f_{4}\left(S_{m}^{n-1}, E_{m}^{n-1}, I_{m}^{n-1}, S_{h}^{n-1}, E_{h}^{n-1}, I_{h}^{n-1}, P_{h}^{n-1}, R_{h}^{n-1}, t_{n-1}\right) \\
& +\frac{\theta_{1} \theta_{2}}{M\left(\theta_{1}\right)} \int_{t_{n}}^{t_{n+1}} \lambda^{\theta_{2}-1} f_{4}\left(S_{m}, E_{m}, I_{m}, S_{h}, E_{h}, I_{h}, P_{h}, R_{h}, \lambda\right) d \lambda, \\
& E_{h}^{n+1}=E_{h}^{n}+\frac{\theta_{2} t^{\theta_{2}-1}\left(1-\theta_{1}\right)}{M\left(\theta_{1}\right)} f_{5}\left(S_{m}^{n}, E_{m}^{n}, I_{m}^{n}, S_{h}^{n}, E_{h}^{n}, I_{h}^{n}, P_{h}^{n}, R_{h}^{n}, t_{n}\right) \\
& -\frac{\theta_{2} t_{n-1}^{\theta_{2}-1}\left(1-\theta_{1}\right)}{M\left(\theta_{1}\right)} f_{5}\left(S_{m}^{n-1}, E_{m}^{n-1}, I_{m}^{n-1}, S_{h}^{n-1}, E_{h}^{n-1}, I_{h}^{n-1}, P_{h}^{n-1}, R_{h}^{n-1}, t_{n-1}\right) \\
& +\frac{\theta_{1} \theta_{2}}{M\left(\theta_{1}\right)} \int_{t_{n}}^{t_{n+1}} \lambda^{\theta_{2}-1} f_{5}\left(S_{m}, E_{m}, I_{m}, S_{h}, E_{h}, I_{h}, P_{h}, R_{h}, \lambda\right) d \lambda, \\
& I_{h}^{n+1}=I_{h}^{n}+\frac{\theta_{2} t^{\theta_{2}-1}\left(1-\theta_{1}\right)}{M\left(\theta_{1}\right)} f_{6}\left(S_{m}^{n}, E_{m}^{n}, I_{m}^{n}, S_{h}^{n}, E_{h}^{n}, I_{h}^{n}, P_{h}^{n}, R_{h}^{n}, t_{n}\right) \\
& -\frac{\theta_{2} t_{n-1}^{\theta_{2}-1}\left(1-\theta_{1}\right)}{M\left(\theta_{1}\right)} f_{6}\left(S_{m}^{n-1}, E_{m}^{n-1}, I_{m}^{n-1}, S_{h}^{n-1}, E_{h}^{n-1}, I_{h}^{n-1}, P_{h}^{n-1}, R_{h}^{n-1}, t_{n-1}\right)
\end{aligned}
$$




$$
\begin{aligned}
& +\frac{\theta_{1} \theta_{2}}{M\left(\theta_{1}\right)} \int_{t_{n}}^{t_{n+1}} \lambda^{\theta_{2}-1} f_{6}\left(S_{m}, E_{m}, I_{m}, S_{h}, E_{h}, I_{h}, P_{h}, R_{h}, \lambda\right) d \lambda, \\
P_{h}^{n+1}= & P_{h}^{n}+\frac{\theta_{2} t^{\theta_{2}-1}\left(1-\theta_{1}\right)}{M\left(\theta_{1}\right)} f_{7}\left(S_{m}^{n}, E_{m}^{n}, I_{m}^{n}, S_{h}^{n}, E_{h}^{n}, I_{h}^{n}, P_{h}^{n}, R_{h}^{n}, t_{h}\right) \\
& -\frac{\theta_{2} t_{n-1}^{\theta_{2}-1}\left(1-\theta_{1}\right)}{M\left(\theta_{1}\right)} f_{7}\left(S_{m}^{n-1}, E_{m}^{n-1}, I_{m}^{n-1}, S_{h}^{n-1}, E_{h}^{n-1}, I_{h}^{n-1}, P_{h}^{n-1}, R_{h}^{n-1}, t_{n-1}\right) \\
& +\frac{\theta_{1} \theta_{2}}{M\left(\theta_{1}\right)} \int_{t_{n}}^{t_{n+1}} \lambda^{\theta_{2}-1} f_{7}\left(S_{m}, E_{m}, I_{m}, S_{h}, E_{h}, I_{h}, P_{h}, R_{h}, \lambda\right) d \lambda, \\
R_{h}^{n+1}= & R_{h}^{n}+\frac{\theta_{2} t^{\theta_{2}-1}\left(1-\theta_{1}\right)}{M\left(\theta_{1}\right)} f_{8}\left(S_{m}^{n}, E_{m}^{n}, I_{m}^{n}, S_{h}^{n}, E_{h}^{n}, I_{h}^{n}, P_{h}^{n}, R_{h}^{n}, t_{h}\right) \\
& -\frac{\theta_{2} t_{n-1}^{\theta_{2}-1}\left(1-\theta_{1}\right)}{M\left(\theta_{1}\right)} f_{8}\left(S_{m}^{n-1}, E_{m}^{n-1}, I_{m}^{n-1}, S_{h}^{n-1}, E_{h}^{n-1}, I_{h}^{n-1}, P_{h}^{n-1}, R_{h}^{n-1}, t_{n-1}\right) \\
& +\frac{\theta_{1} \theta_{2}}{M\left(\theta_{1}\right)} \int_{t_{n}}^{t_{n+1}} \lambda^{\theta_{2}-1} f_{8}\left(S_{m}, E_{m}, I_{m}, S_{h}, E_{h}, I_{h}, P_{h}, R_{h}, \lambda\right) d \lambda .
\end{aligned}
$$

It follows from the Lagrange polynomial interpolation and integrating the following expressions that

$$
\begin{aligned}
S_{m}^{n+1}= & S_{m}^{n}+\frac{\theta_{2} t^{\theta_{2}-1}\left(1-\theta_{1}\right)}{M\left(\theta_{1}\right)} f_{1}\left(S_{m}^{n}, E_{m}^{n}, I_{m}^{n}, S_{h}^{n}, E_{h}^{n}, I_{h}^{n}, P_{h}^{n}, R_{h}^{n}, t_{n}\right) \\
& -\frac{\theta_{2} t_{n-1}^{\theta_{2}-1}\left(1-\theta_{1}\right)}{M\left(\theta_{1}\right)} f_{1}\left(S_{m}^{n-1}, E_{m}^{n-1}, I_{m}^{n-1}, S_{h}^{n-1}, E_{h}^{n-1}, I_{h}^{n-1}, P_{h}^{n-1}, R_{h}^{n-1}, t_{n-1}\right) \\
& +\frac{\theta_{1} \theta_{2}}{M\left(\theta_{1}\right)}\left[\frac{3 h}{2} t_{n}^{\theta_{2}-1} f_{1}\left(S_{m}^{n}, E_{m}^{n}, I_{m}^{n}, S_{h}^{n}, E_{h}^{n}, I_{h}^{n}, P_{h}^{n}, R_{h}^{n}, t_{n}\right)\right. \\
& \left.-\frac{h}{2} t_{n-1}^{\theta_{2}-1} f_{1}\left(S_{m}^{n-1}, E_{m}^{n-1}, I_{m}^{n-1}, S_{h}^{n-1}, E_{h}^{n-1}, I_{h}^{n-1}, P_{h}^{n-1}, R_{h}^{n-1}, t_{n-1}\right)\right], \\
E_{m}^{n+1}= & E_{m}^{n}+\frac{\theta_{2} t^{\theta_{2}-1}\left(1-\theta_{1}\right)}{M\left(\theta_{1}\right)} f_{2}\left(S_{m}^{n}, E_{m}^{n}, I_{m}^{n}, S_{h}^{n}, E_{h}^{n}, I_{h}^{n}, P_{h}^{n}, R_{h}^{n}, t_{n}\right) \\
& -\frac{\theta_{2} t_{n-1}^{\theta_{2}-1}\left(1-\theta_{1}\right)}{M\left(\theta_{1}\right)} f_{2}\left(S_{m}^{n-1}, E_{m}^{n-1}, I_{m}^{n-1}, S_{h}^{n-1}, E_{h}^{n-1}, I_{h}^{n-1}, P_{h}^{n-1}, R_{h}^{n-1}, t_{n-1}\right) \\
& +\frac{\theta_{1} \theta_{2}}{M\left(\theta_{1}\right)}\left[\frac{3 h}{2} t_{n}^{\theta_{2}-1} f_{2}\left(S_{m}^{n}, E_{m}^{n}, I_{m}^{n}, S_{h}^{n}, E_{h}^{n}, I_{h}^{n}, P_{h}^{n}, R_{h}^{n}, t_{n}\right)\right. \\
& \left.-\frac{h}{2} t_{n-1}^{\theta_{2}-1} f_{2}\left(S_{m}^{n-1}, E_{m}^{n-1}, I_{m}^{n-1}, S_{h}^{n-1}, E_{h}^{n-1}, I_{h}^{n-1}, P_{h}^{n-1}, R_{h}^{n-1}, t_{n-1}\right)\right], \\
S_{h}^{n+1}= & S_{h}^{n}+\frac{\theta_{2} t^{\theta_{2}-1}\left(1-\theta_{1}\right)}{M\left(\theta_{1}\right)} f_{4}\left(S_{m}^{n}, E_{m}^{n}, I_{m}^{n}, S_{h}^{n}, E_{h}^{n}, I_{h}^{n}, P_{h}^{n}, R_{h}^{n}, t_{n}\right) \\
I_{m}^{n+1}= & I_{m}^{n}+\frac{\theta_{2} t^{\theta_{2}-1}\left(1-\theta_{1}\right)}{M\left(\theta_{1}\right)} f_{3}\left(S_{m}^{n}, E_{m}^{n}, I_{m}^{n}, S_{h}^{n}, E_{h}^{n}, I_{h}^{n}, P_{h}^{n}, R_{h}^{n}, t_{n}\right) \\
& -\frac{\theta_{2} t_{n_{2}-1}^{\theta_{2}-1}\left(1-\theta_{1}\right)}{M\left(\theta_{1}\right)} f_{3}\left(S_{m}^{n-1}, E_{m}^{n-1}, I_{m}^{n-1}, S_{h}^{n-1}, E_{h}^{n-1}, I_{h}^{n-1}, P_{h}^{n-1}, R_{h}^{n-1}, t_{n-1}\right) \\
& +\frac{\theta_{1} \theta_{2}}{M\left(\theta_{1}\right)}\left[\frac{3 h}{2} t_{n}^{\theta_{2}-1} f_{3}\left(S_{m}^{n}, E_{m}^{n}, I_{m}^{n}, S_{h}^{n}, E_{h}^{n}, I_{h}^{n}, P_{h}^{n}, R_{h}^{n}, t_{n}\right)\right. \\
& \left.-\frac{h}{\theta_{2}-1} f_{3}\left(S_{m}^{n-1}, E_{m}^{n-1}, I_{m}^{n-1}, S_{h}^{n-1}, E_{h}^{n-1}, I_{h}^{n-1}, P_{h}^{n-1}, R_{h}^{n-1}, t_{n-1}\right)\right], \\
&
\end{aligned}
$$




$$
\begin{aligned}
& -\frac{\theta_{2} t_{n-1}^{\theta_{2}-1}\left(1-\theta_{1}\right)}{M\left(\theta_{1}\right)} f_{4}\left(S_{m}^{n-1}, E_{m}^{n-1}, I_{m}^{n-1}, S_{h}^{n-1}, E_{h}^{n-1}, I_{h}^{n-1}, P_{h}^{n-1}, R_{h}^{n-1}, t_{n-1}\right) \\
& +\frac{\theta_{1} \theta_{2}}{M\left(\theta_{1}\right)}\left[\frac{3 h}{2} t_{n}^{\theta_{2}-1} f_{4}\left(S_{m}^{n}, E_{m}^{n}, I_{m}^{n}, S_{h}^{n}, E_{h}^{n}, I_{h}^{n}, P_{h}^{n}, R_{h}^{n}, t_{n}\right)\right. \\
& \left.-\frac{h}{2} t_{n-1}^{\theta_{2}-1} f_{4}\left(S_{m}^{n-1}, E_{m}^{n-1}, I_{m}^{n-1}, S_{h}^{n-1}, E_{h}^{n-1}, I_{h}^{n-1}, P_{h}^{n-1}, R_{h}^{n-1}, t_{n-1}\right)\right], \\
& E_{h}^{n+1}=E_{h}^{n}+\frac{\theta_{2} t^{\theta_{2}-1}\left(1-\theta_{1}\right)}{M\left(\theta_{1}\right)} f_{5}\left(S_{m}^{n}, E_{m}^{n}, I_{m}^{n}, S_{h}^{n}, E_{h}^{n}, I_{h}^{n}, P_{h}^{n}, R_{h}^{n}, t_{n}\right) \\
& -\frac{\theta_{2} t_{n-1}^{\theta_{2}-1}\left(1-\theta_{1}\right)}{M\left(\theta_{1}\right)} f_{5}\left(S_{m}^{n-1}, E_{m}^{n-1}, I_{m}^{n-1}, S_{h}^{n-1}, E_{h}^{n-1}, I_{h}^{n-1}, P_{h}^{n-1}, R_{h}^{n-1}, t_{n-1}\right) \\
& +\frac{\theta_{1} \theta_{2}}{M\left(\theta_{1}\right)}\left[\frac{3 h}{2} t_{n}^{\theta_{2}-1} f_{5}\left(S_{m}^{n}, E_{m}^{n}, I_{m}^{n}, S_{h}^{n}, E_{h}^{n}, I_{h}^{n}, P_{h}^{n}, R_{h}^{n}, t_{n}\right)\right. \\
& \left.-\frac{h}{2} t_{n-1}^{\theta_{2}-1} f_{5}\left(S_{m}^{n-1}, E_{m}^{n-1}, I_{m}^{n-1}, S_{h}^{n-1}, E_{h}^{n-1}, I_{h}^{n-1}, P_{h}^{n-1}, R_{h}^{n-1}, t_{n-1}\right)\right] \\
& I_{h}^{n+1}=I_{h}^{n}+\frac{\theta_{2} t^{\theta_{2}-1}\left(1-\theta_{1}\right)}{M\left(\theta_{1}\right)} f_{6}\left(S_{m}^{n}, E_{m}^{n}, I_{m}^{n}, S_{h}^{n}, E_{h}^{n}, I_{h}^{n}, P_{h}^{n}, R_{h}^{n}, t_{n}\right) \\
& -\frac{\theta_{2} t_{n-1}^{\theta_{2}-1}\left(1-\theta_{1}\right)}{M\left(\theta_{1}\right)} f_{6}\left(S_{m}^{n-1}, E_{m}^{n-1}, I_{m}^{n-1}, S_{h}^{n-1}, E_{h}^{n-1}, I_{h}^{n-1}, P_{h}^{n-1}, R_{h}^{n-1}, t_{n-1}\right) \\
& +\frac{\theta_{1} \theta_{2}}{M\left(\theta_{1}\right)}\left[\frac{3 h}{2} t_{n}^{\theta_{2}-1} f_{6}\left(S_{m}^{n}, E_{m}^{n}, I_{m}^{n}, S_{h}^{n}, E_{h}^{n}, I_{h}^{n}, P_{h}^{n}, R_{h}^{n}, t_{n}\right)\right. \\
& \left.-\frac{h}{2} t_{n-1}^{\theta_{2}-1} f_{6}\left(S_{m}^{n-1}, E_{m}^{n-1}, I_{m}^{n-1}, S_{h}^{n-1}, E_{h}^{n-1}, I_{h}^{n-1}, P_{h}^{n-1}, R_{h}^{n-1}, t_{n-1}\right)\right] \text {, } \\
& P_{h}^{n+1}=P_{h}^{n}+\frac{\theta_{2} t^{\theta_{2}-1}\left(1-\theta_{1}\right)}{M\left(\theta_{1}\right)} f_{7}\left(S_{m}^{n}, E_{m}^{n}, I_{m}^{n}, S_{h}^{n}, E_{h}^{n}, I_{h}^{n}, P_{h}^{n}, R_{h}^{n}, t_{n}\right) \\
& -\frac{\theta_{2} t_{n-1}^{\theta_{2}-1}\left(1-\theta_{1}\right)}{M\left(\theta_{1}\right)} f_{7}\left(S_{m}^{n-1}, E_{m}^{n-1}, I_{m}^{n-1}, S_{h}^{n-1}, E_{h}^{n-1}, I_{h}^{n-1}, P_{h}^{n-1}, R_{h}^{n-1}, t_{n-1}\right) \\
& +\frac{\theta_{1} \theta_{2}}{M\left(\theta_{1}\right)}\left[\frac{3 h}{2} t_{n}^{\theta_{2}-1} f_{7}\left(S_{m}^{n}, E_{m}^{n}, I_{m}^{n}, S_{h}^{n}, E_{h}^{n}, I_{h}^{n}, P_{h}^{n}, R_{h}^{n}, t_{n}\right)\right. \\
& \left.-\frac{h}{2} t_{n-1}^{\theta_{2}-1} f_{7}\left(S_{m}^{n-1}, E_{m}^{n-1}, I_{m}^{n-1}, S_{h}^{n-1}, E_{h}^{n-1}, I_{h}^{n-1}, P_{h}^{n-1}, R_{h}^{n-1}, t_{n-1}\right)\right] \text {, } \\
& R_{h}^{n+1}=R_{h}^{n}+\frac{\theta_{2} t^{\theta_{2}-1}\left(1-\theta_{1}\right)}{M\left(\theta_{1}\right)} f_{8}\left(S_{m}^{n}, E_{m}^{n}, I_{m}^{n}, S_{h}^{n}, E_{h}^{n}, I_{h}^{n}, P_{h}^{n}, R_{h}^{n}, t_{n}\right) \\
& -\frac{\theta_{2} t_{n-1}^{\theta_{2}-1}\left(1-\theta_{1}\right)}{M\left(\theta_{1}\right)} f_{8}\left(S_{v}^{n-1}, E_{v}^{n-1}, I_{v}^{n-1}, S_{h}^{n-1}, E_{h}^{n-1}, I_{h}^{n-1}, P_{h}^{n-1}, R_{h}^{n-1}, t_{n-1}\right) \\
& +\frac{\theta_{1} \theta_{2}}{M\left(\theta_{1}\right)}\left[\frac{3 h}{2} t_{n}^{\theta_{2}-1} f_{8}\left(S_{m}^{n}, E_{m}^{n}, I_{m}^{n}, S_{h}^{n}, E_{h}^{n}, I_{h}^{n}, P_{h}^{n}, R_{h}^{n}, t_{n}\right)\right. \\
& \left.-\frac{h}{2} t_{n-1}^{\theta_{2}-1} f_{8}\left(S_{m}^{n-1}, E_{m}^{n-1}, I_{m}^{n-1}, S_{h}^{n-1}, E_{h}^{n-1}, I_{h}^{n-1}, P_{h}^{n-1}, R_{h}^{n-1}, t_{n-1}\right)\right] \text {. }
\end{aligned}
$$

Further simplifications of (18) lead to the following:

$$
\begin{aligned}
S_{m}^{n+1}= & S_{m}^{n}+\theta_{2} t_{n}^{\theta_{2}-1}\left(\frac{1-\theta_{1}}{M\left(\theta_{1}\right)}+\frac{3 \theta_{1} h}{2 M\left(\theta_{1}\right)}\right) f_{1}\left(S_{m}^{n}, E_{m}^{n}, I_{m}^{n}, S_{h}^{n}, E_{h}^{n}, I_{h}^{n}, P_{h}^{n}, R_{h}^{n}, t_{n}\right) \\
& -\theta_{2} t_{n-1}^{\theta_{2}-1}\left(\frac{1-\theta_{1}}{M\left(\theta_{1}\right)}+\frac{\theta_{1} h}{2 M\left(\theta_{1}\right)}\right)
\end{aligned}
$$




$$
\begin{aligned}
& \times f_{1}\left(S_{m}^{n-1}, E_{m}^{n-1}, I_{m}^{n-1}, S_{h}^{n-1}, E_{h}^{n-1}, I_{h}^{n-1}, P_{h}^{n-1}, R_{h}^{n-1}, t_{n-1}\right), \\
& E_{m}^{n+1}=E_{m}^{n}+\theta_{2} t_{n}^{\theta_{2}-1}\left(\frac{1-\theta_{1}}{M\left(\theta_{1}\right)}+\frac{3 \theta_{1} h}{2 M\left(\theta_{1}\right)}\right) f_{2}\left(S_{m}^{n}, E_{m}^{n}, I_{m}^{n}, S_{h}^{n}, E_{h}^{n}, I_{h}^{n}, P_{h}^{n}, R_{h}^{n}, t_{n}\right) \\
& -\theta_{2} t_{n-1}^{\theta_{2}-1}\left(\frac{1-\theta_{1}}{M\left(\theta_{1}\right)}+\frac{\theta_{1} h}{2 M\left(\theta_{1}\right)}\right) \\
& \times f_{2}\left(S_{m}^{n-1}, E_{m}^{n-1}, I_{m}^{n-1}, S_{h}^{n-1}, E_{h}^{n-1}, I_{h}^{n-1}, P_{h}^{n-1}, R_{h}^{n-1}, t_{n-1}\right), \\
& I_{m}^{n+1}=I_{m}^{n}+\theta_{2} t_{n}^{\theta_{2}-1}\left(\frac{1-\theta_{1}}{M\left(\theta_{1}\right)}+\frac{3 \theta_{1} h}{2 M\left(\theta_{1}\right)}\right) f_{3}\left(S_{m}^{n}, E_{m}^{n}, I_{m}^{n}, S_{h}^{n}, E_{h}^{n}, I_{h}^{n}, P_{h}^{n}, R_{h}^{n}, t_{n}\right) \\
& -\theta_{2} t_{n-1}^{\theta_{2}-1}\left(\frac{1-\theta_{1}}{M\left(\theta_{1}\right)}+\frac{\theta_{1} h}{2 M\left(\theta_{1}\right)}\right) \\
& \times f_{3}\left(S_{m}^{n-1}, E_{m}^{n-1}, I_{m}^{n-1}, S_{h}^{n-1}, E_{h}^{n-1}, I_{h}^{n-1}, P_{h}^{n-1}, R_{h}^{n-1}, t_{n-1}\right), \\
& S_{h}^{n+1}=S_{h}^{n}+\theta_{2} t_{n}^{\theta_{2}-1}\left(\frac{1-\theta_{1}}{M\left(\theta_{1}\right)}+\frac{3 \theta_{1} h}{2 M\left(\theta_{1}\right)}\right) f_{4}\left(S_{m}^{n}, E_{m}^{n}, I_{m}^{n}, S_{h}^{n}, E_{h}^{n}, I_{h}^{n}, P_{h}^{n}, R_{h}^{n}, t_{n}\right) \\
& -\theta_{2} t_{n-1}^{\theta_{2}-1}\left(\frac{1-\theta_{1}}{M\left(\theta_{1}\right)}+\frac{\theta_{1} h}{2 M\left(\theta_{1}\right)}\right) \\
& \times f_{4}\left(S_{m}^{n-1}, E_{m}^{n-1}, I_{m}^{n-1}, S_{h}^{n-1}, E_{h}^{n-1}, I_{h}^{n-1}, P_{h}^{n-1}, R_{h}^{n-1}, t_{n-1}\right), \\
& E_{h}^{n+1}=E_{h}^{n}+\theta_{2} t_{n}^{\theta_{2}-1}\left(\frac{1-\theta_{1}}{M\left(\theta_{1}\right)}+\frac{3 \theta_{1} h}{2 M\left(\theta_{1}\right)}\right) f_{5}\left(S_{m}^{n}, E_{m}^{n}, I_{m}^{n}, S_{h}^{n}, E_{h}^{n}, I_{h}^{n}, P_{h}^{n}, R_{h}^{n}, t_{n}\right) \\
& -\theta_{2} t_{n-1}^{\theta_{2}-1}\left(\frac{1-\theta_{1}}{M\left(\theta_{1}\right)}+\frac{\theta_{1} h}{2 M\left(\theta_{1}\right)}\right) \\
& \times f_{5}\left(S_{m}^{n-1}, E_{m}^{n-1}, I_{m}^{n-1}, S_{h}^{n-1}, E_{h}^{n-1}, I_{h}^{n-1}, P_{h}^{n-1}, R_{h}^{n-1}, t_{n-1}\right), \\
& I_{h}^{n+1}=I_{h}^{n}+\theta_{2} t_{n}^{\theta_{2}-1}\left(\frac{1-\theta_{1}}{M\left(\theta_{1}\right)}+\frac{3 \theta_{1} h}{2 M\left(\theta_{1}\right)}\right) f_{6}\left(S_{m}^{n}, E_{m}^{n}, I_{m}^{n}, S_{h}^{n}, E_{h}^{n}, I_{h}^{n}, P_{h}^{n}, R_{h}^{n}, t_{n}\right) \\
& -\theta_{2} t_{n-1}^{\theta_{2}-1}\left(\frac{1-\theta_{1}}{M\left(\theta_{1}\right)}+\frac{\theta_{1} h}{2 M\left(\theta_{1}\right)}\right) \\
& \times f_{6}\left(S_{m}^{n-1}, E_{m}^{n-1}, I_{m}^{n-1}, S_{h}^{n-1}, E_{h}^{n-1}, I_{h}^{n-1}, P_{h}^{n-1}, R_{h}^{n-1}, t_{n-1}\right), \\
& P_{h}^{n+1}=P_{h}^{n}+\theta_{2} t_{n}^{\theta_{2}-1}\left(\frac{1-\theta_{1}}{M\left(\theta_{1}\right)}+\frac{3 \theta_{1} h}{2 M\left(\theta_{1}\right)}\right) f_{7}\left(S_{m}^{n}, E_{m}^{n}, I_{m}^{n}, S_{h}^{n}, E_{h}^{n}, I_{h}^{n}, P_{h}^{n}, R_{h}^{n}, t_{n}\right) \\
& -\theta_{2} t_{n-1}^{\theta_{2}-1}\left(\frac{1-\theta_{1}}{M\left(\theta_{1}\right)}+\frac{\theta_{1} h}{2 M\left(\theta_{1}\right)}\right) \\
& \times f_{7}\left(S_{v}^{n-1}, E_{v}^{n-1}, I_{v}^{n-1}, S_{h}^{n-1}, E_{h}^{n-1}, I_{h}^{n-1}, P_{h}^{n-1}, R_{h}^{n-1}, t_{n-1}\right), \\
& R_{h}^{n+1}=R_{h}^{n}+\theta_{2} t_{n}^{\theta_{2}-1}\left(\frac{1-\theta_{1}}{M\left(\theta_{1}\right)}+\frac{3 \theta_{1} h}{2 M\left(\theta_{1}\right)}\right) f_{8}\left(S_{m}^{n}, E_{m}^{n}, I_{m}^{n}, S_{h}^{n}, E_{h}^{n}, I_{h}^{n}, P_{h}^{n}, R_{h}^{n}, t_{n}\right) \\
& -\theta_{2} t_{n-1}^{\theta_{2}-1}\left(\frac{1-\theta_{1}}{M\left(\theta_{1}\right)}+\frac{\theta_{1} h}{2 M\left(\theta_{1}\right)}\right) \\
& \times f_{8}\left(S_{m}^{n-1}, E_{m}^{n-1}, I_{m}^{n-1}, S_{h}^{n-1}, E_{h}^{n-1}, I_{h}^{n-1}, P_{h}^{n-1}, R_{h}^{n-1}, t_{n-1}\right) .
\end{aligned}
$$

\section{Numerical results}

In order to graphically illustrate the numerical results for the fractal-fractional model in the sense of Caputo-Fabrizio operator, we first estimate the model parameters and the initial conditions for the model. We present the following subsection for parameter estimates. 


\subsection{Parameter estimation of dengue model}

In the present section, we estimate the parameters of the model (10) for the case when $\theta_{1}=\theta_{2}=1$ of dengue fever cases in East Java, Indonesia, for the year 2018. Based on data obtained from the East Java provincial health office, it was reported that the incidence rate of Dengue Hemorrhagic Fever (DHF) in East Java in 2016 was 64.8 per 100,000 people, an increase compared to 2015 which was 54.18 per 100,000 people, while the incidence rate of DHF in 2017 was 20 per 100,000 people [37]. Although in 2017 the number of cases of DHF decreased compared to the previous year, the awareness of the surge in cases in the next year needs to be improved. In 2017, the total number of DHF in East Java reached 7854 people, while in 2018 it reached 9452 people [38]. From this, it appears that there is an increase in the number of DHF cases in 2018 compared to 2017. Hence, the cumulative monthly reported DHF cases from January to December 2018 are used to parameterize the model (10). In order to get a good fit to the real data, we estimate the parameters using the least square curve fitting technique except for the recruitment rate of human $\Pi_{h}$ and the natural death rate of human $v_{h}$. The parameter $v_{h}$ is calculated as the inverse of the average lifespan in East Java so that $v_{h}=1 / 70.97$ per year, where 70.97 year is the average lifespan population in East Java province [39]. The parameter $\Pi_{h}$ is computed as follows. Since the total population of East Java province as of 2018 was 39,507,370, we have that $\Pi_{h} / v_{h}=39,507,370$ is the maximum human population without the disease, therefore $\Pi_{h}=556,677.0466$ per year. The other parameters are obtained using the least-squares curve fitting method. The fitted and estimated parameter values of the model (10) are listed in Table 2. The result of fitting the model (10) to the actual data of dengue incidence is displayed in Fig. 1. Using the parameter values in Table 2, the basic reproduction number in East Java is $\mathcal{R}_{0} \approx 2.2020$. The parameters listed in Table 2 are used further to obtain the graphical results for the fractal-fractional model (10).

\subsection{Simulation results}

In this subsection, we apply the novel approach considered above for the fractal-fractional model of dengue in the sense of Caputo-Fabrizio operator to illustrate its numerical results graphically. Throughout these simulation results, the subgraphs in Figs. 2, 4, 6,8 , which represent respectively the dynamics of susceptible, exposed, and infected mosquitos and the susceptible humans, while the subgraphs in Figs. 3, 5, 7, 9 show respectively the components of the exposed, infected, hospitalized, and recovered humans.

Table 2 Fitted and estimated values for the parameters of the model (10)

\begin{tabular}{llcl}
\hline Parameter & Units & Baselines value & References \\
\hline$\Pi_{m}$ & month $^{-1}$ & 3839.9 & Fitted \\
$\tau$ & month $^{-1}$ & 0.9961 & Fitted \\
$\alpha_{h}$ & - & 0.6341 & Fitted \\
$\mu_{m}$ & month $^{-1}$ & $1 / 42$ & {$[12,40]$} \\
$\beta_{m}$ & month $^{-1}$ & 0.586 & Fitted \\
$\Pi_{h}$ & month $^{-1}$ & 46390 & Estimated \\
$\alpha_{v}$ & - & 0.989 & Fitted \\
$\beta_{h}$ & month $^{-1}$ & 0.8850 & Fitted \\
$\psi_{h}$ & month $^{-1}$ & 0.0184 & Fitted \\
$\omega_{h}$ & month $^{-1}$ & 0.00154 & Fitted \\
$\sigma_{h}$ & month $^{-1}$ & 0.440 & Fitted \\
$\tau_{h}$ & month $^{-1}$ & 0.0769 & Fitted \\
$\nu_{h}$ & month $^{-1}$ & $\frac{1}{(70.97 \times 12)}$ & Estimated \\
\hline
\end{tabular}




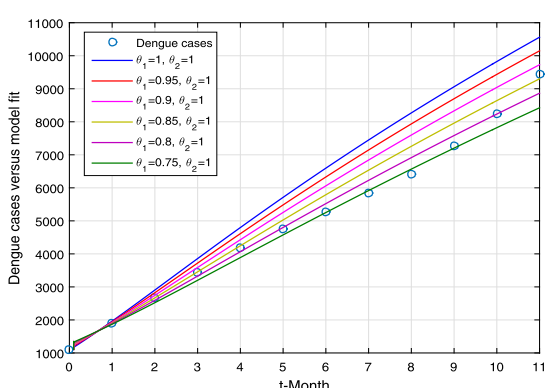

(a)

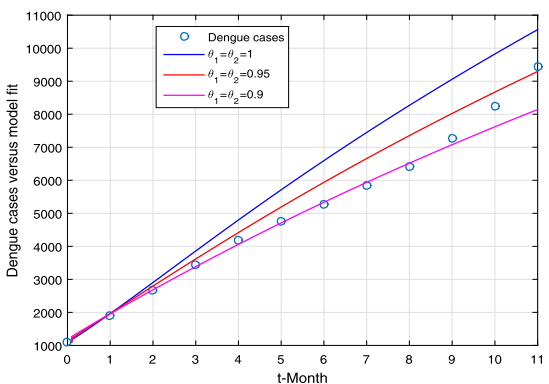

(c)

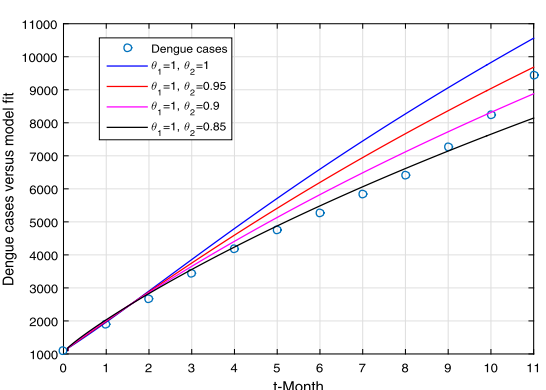

(b)

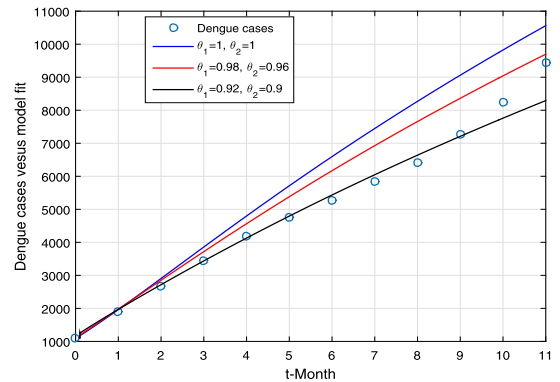

(d)

Figure 1 Model versus data fitting for different values of fractional order $\theta_{1}$ and fractal order $\theta_{2}$

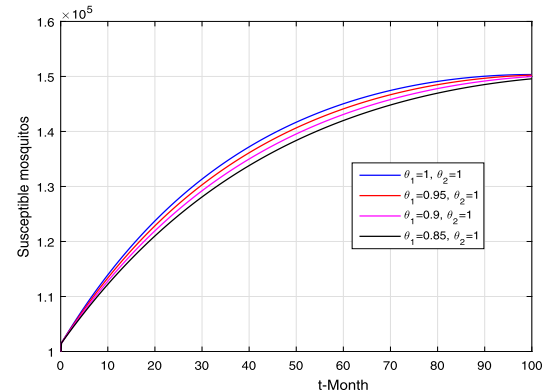

(a)

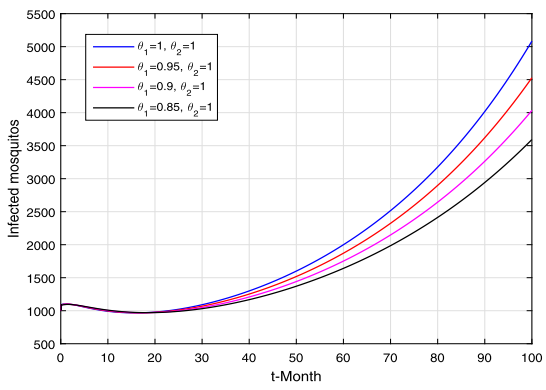

(c)

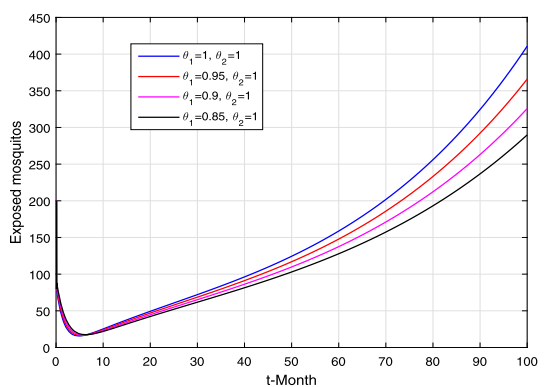

(b)

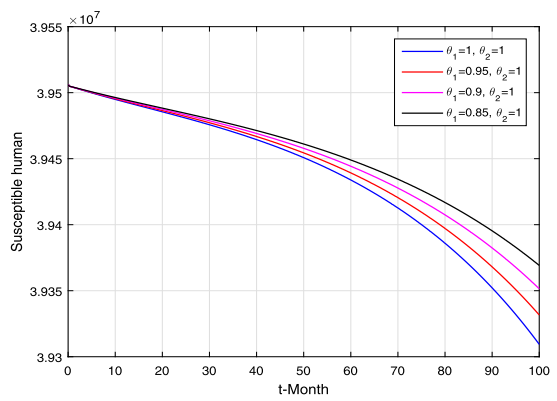

(d)

Figure 2 Simulation of the fractal-fractional model (10) when $\theta_{1}=1,0.95,0.9,0.85$ and fractal order $\theta_{2}=1$ where (a) susceptible mosquitos, (b) exposed mosquitos, (c) infected mosquitos, (d) susceptible humans 


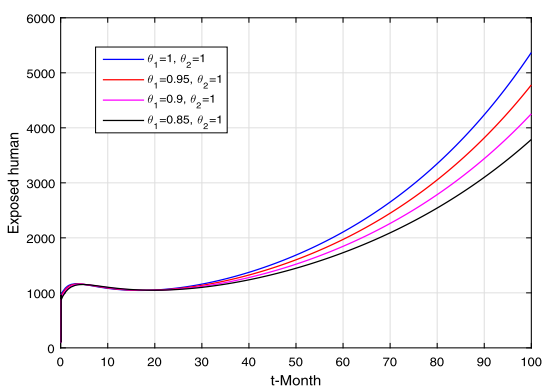

(a)

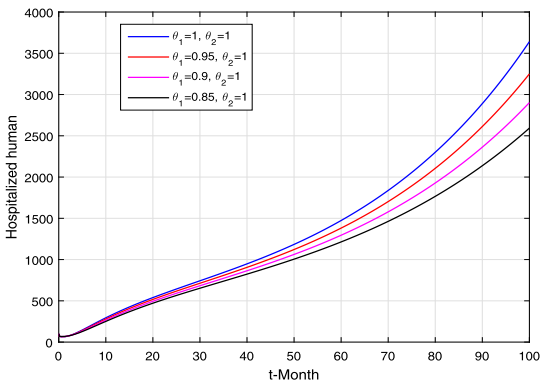

(c)

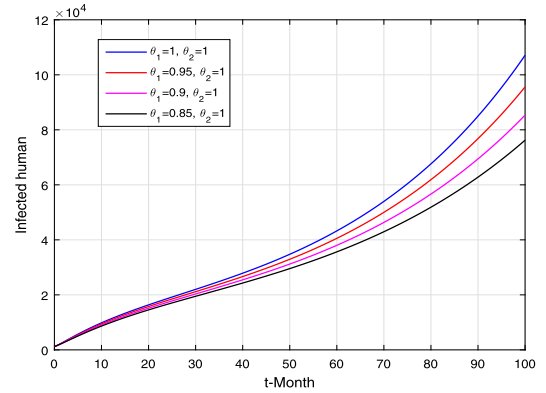

(b)

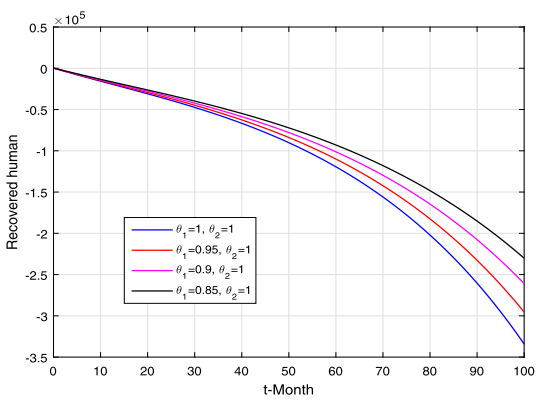

(d)

Figure 3 Simulation of the fractal-fractional model (10) when $\theta_{1}=1,0.95,0.9,0.85$ and fractal order $\theta_{2}=1$ where (a) exposed humans, (b) infected humans, (c) hospitalized humans, (d) recovered humans

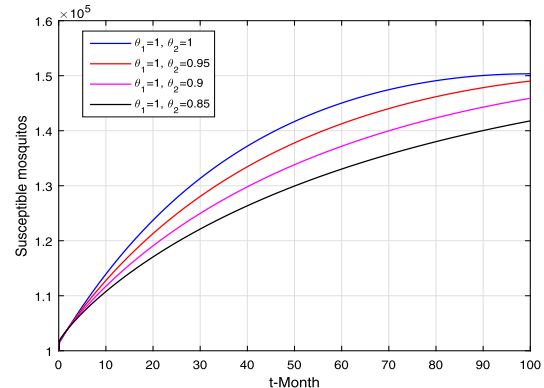

(a)

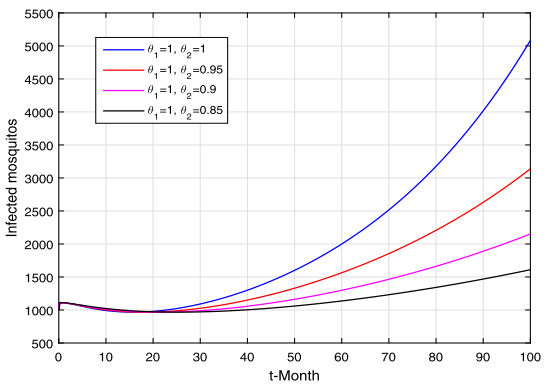

(c)

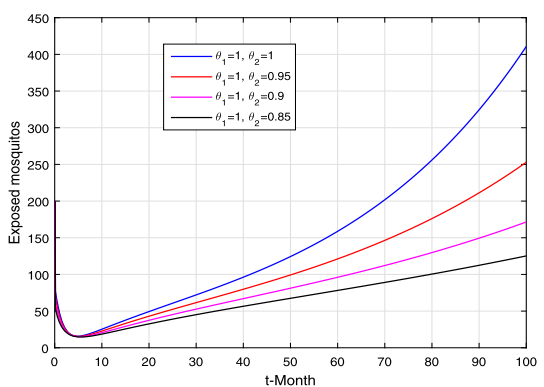

(b)

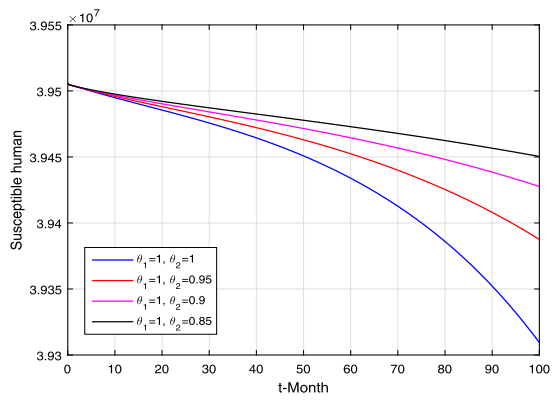

(d)

Figure 4 Simulation of the fractal-fractional model (10) when $\theta_{2}=1,0.95,0.9,0.85$ and fractional order $\theta_{1}=1$ where (a) susceptible mosquitos, (b) exposed mosquitos, (c) infected mosquitos, (d) susceptible humans 


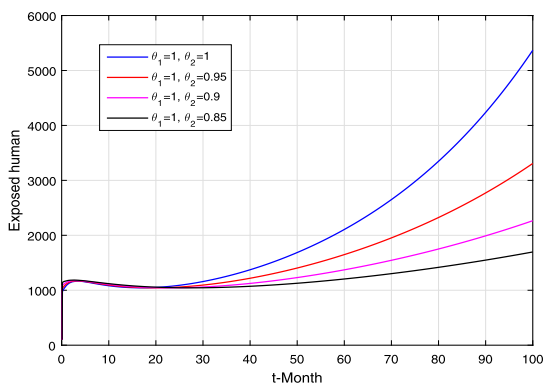

(a)

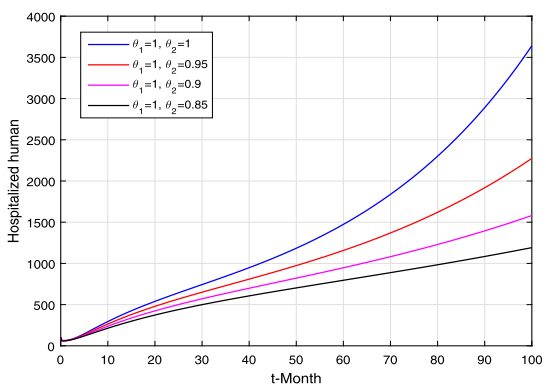

(c)

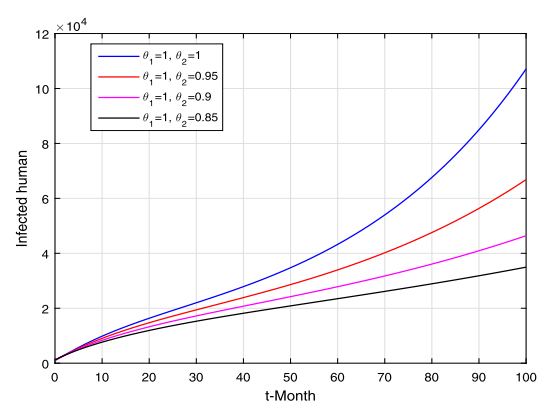

(b)

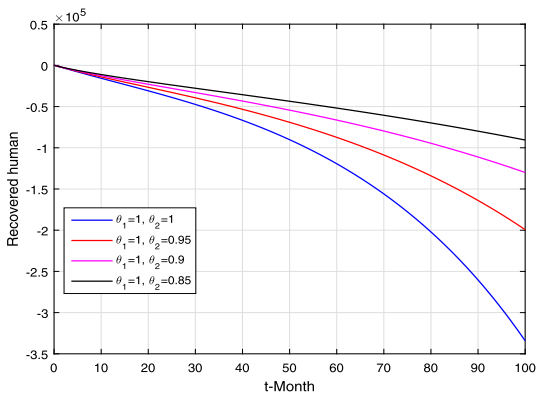

(d)

Figure 5 Simulation of the fractal-fractional model (10) when $\theta_{2}=1,0.95,0.9,0.85$ and fractional order $\theta_{1}=1$ where (a) exposed humans, (b) infected humans, (c) hospitalized humans, (d) recovered humans

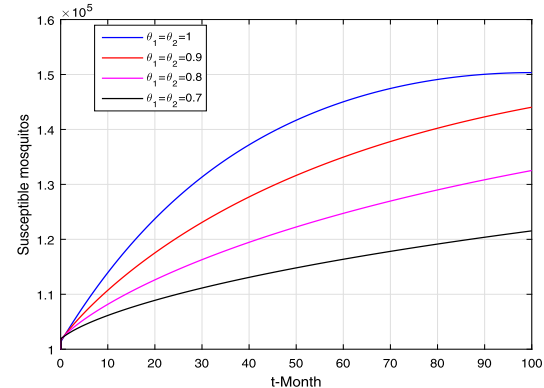

(a)

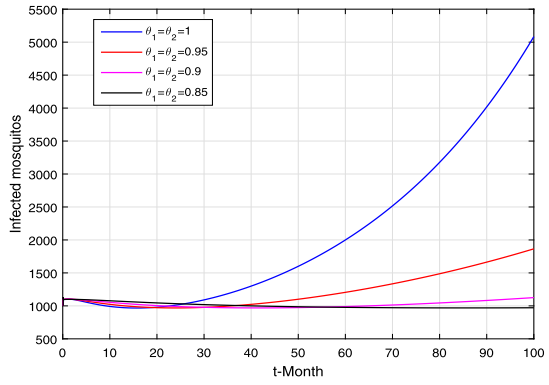

(c)

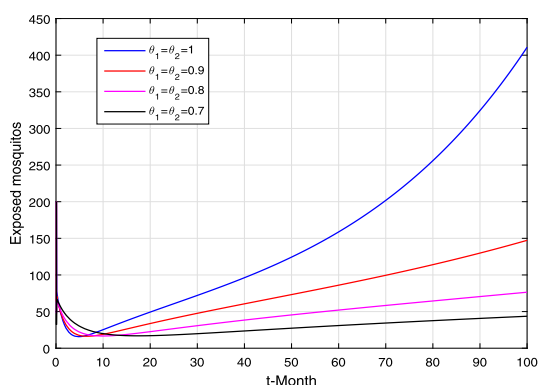

(b)

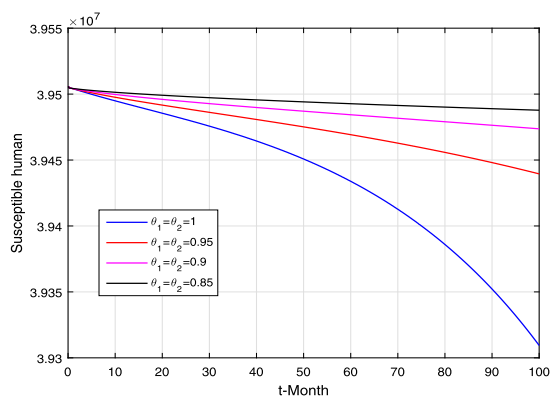

(d)

Figure 6 Simulation of the fractal-fractional model (10) when $\theta_{1}=\theta_{2}=1,0.9,0.8,0.7$ where (a) susceptible mosquitos, (b) exposed mosquitos, (c) infected mosquitos, (d) susceptible humans 


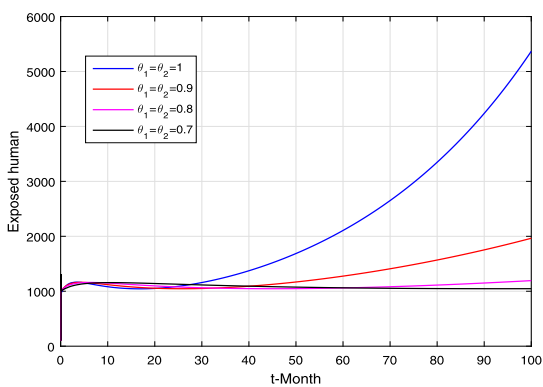

(a)

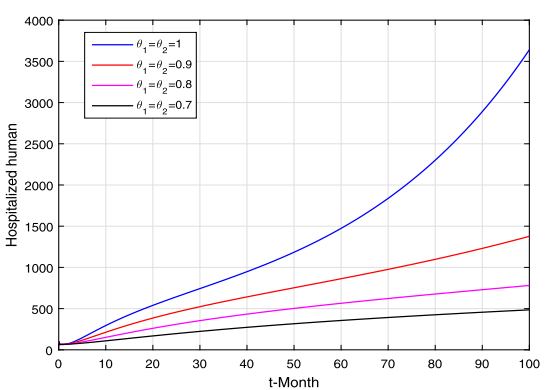

(c)

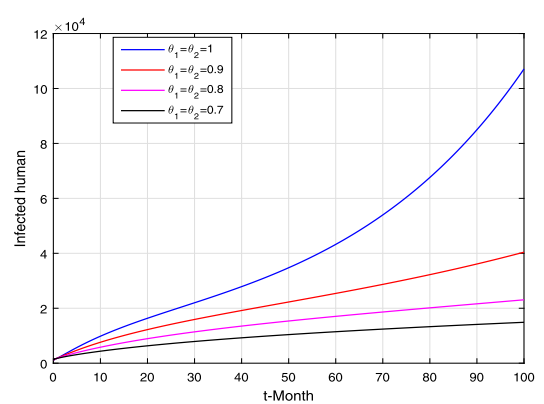

(b)

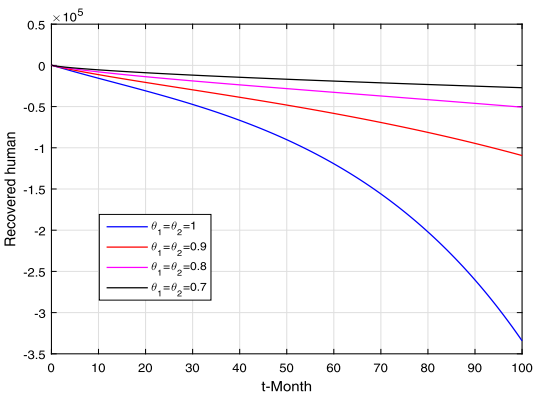

(d)

Figure 7 Simulation of the fractal-fractional model (10) when $\theta_{1}=\theta_{2}=1,0.9,0.8,0.7$ where (a) exposed humans, (b) infected humans, (c) hospitalized humans, (d) recovered humans

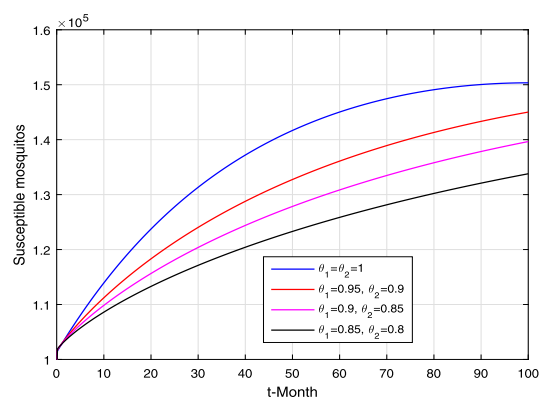

(a)

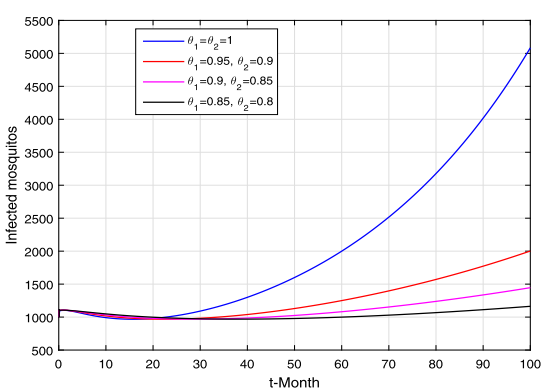

(c)

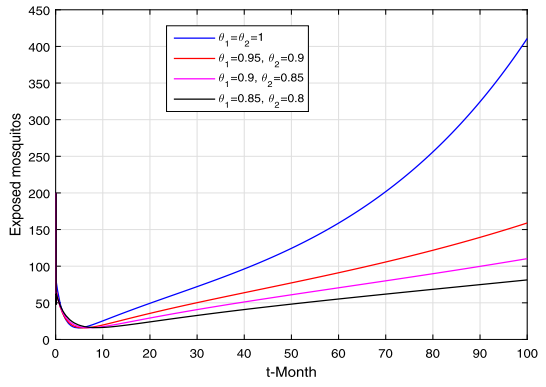

(b)

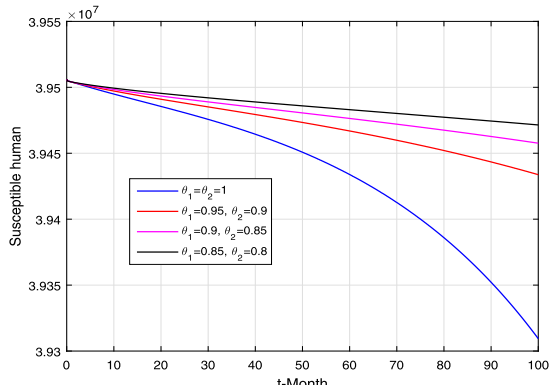

(d)

Figure 8 Simulation of the fractal-fractional model (10) when $\theta_{1}=1,0.95,0.9,0.85$ and fractal order $\theta_{2}=1,0.9,0.85$, 0.8 where (a) susceptible mosquitos, (b) exposed mosquitos, (c) infected mosquitos, (d) susceptible humans 


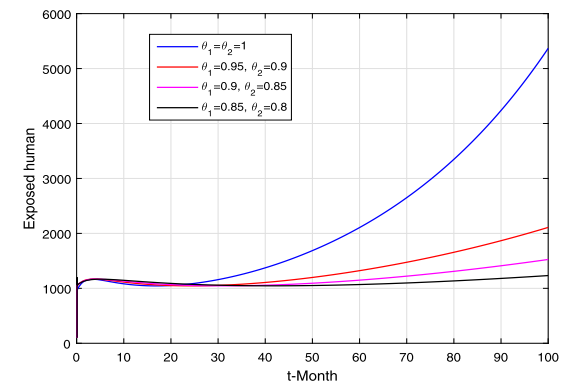

(a)

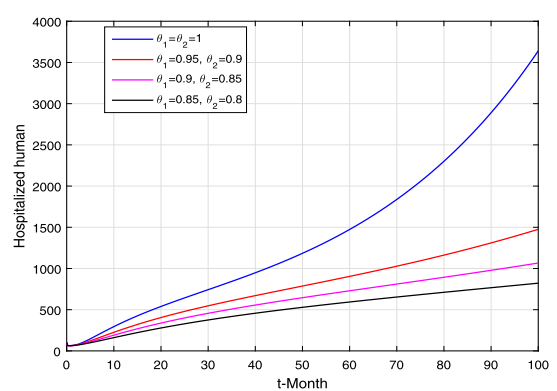

(c)

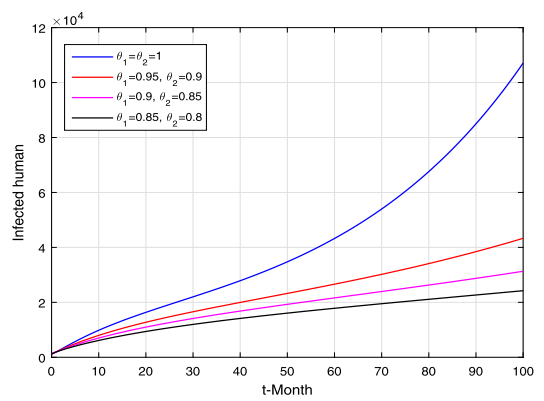

(b)

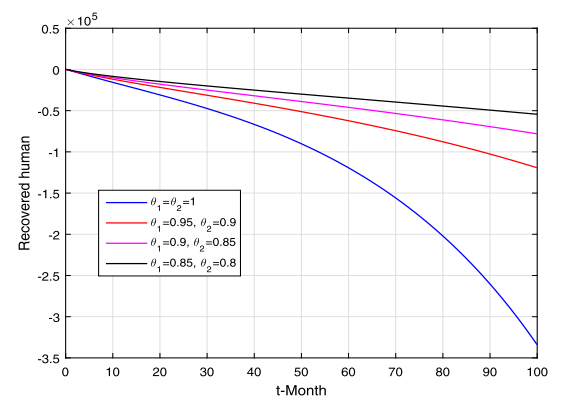

(d)

Figure 9 Simulation of the fractal-fractional model (10) when $\theta_{1}=1,0.95,0.9,0.85$ and fractal order $\theta_{2}=1,0.9,0.85,0.8$ where (a) exposed humans, (b) infected humans, (c) hospitalized humans, (d) recovered humans

We use the estimated and fitted parameters in Table 2 for the simulation results and the units are taken in months. We present the graphical results using the fractal and fractional order parameters $\theta_{1}$ and $\theta_{2}$ in different scenarios. Initially, we choose $\theta_{1}$ as varying, fix the fractal order $\theta_{2}$ and obtain the graphical results shown in Figs. 2 and 3. We obtain Figs. 4 and 5 by choosing the fractal order as varying and fixing the fractional order. Figures 6 and 7 are obtained by setting equal values to the fractional and fractal orders, $\theta_{1}$ and $\theta_{2}$. Figures 7 and 9 are presented for different orders of fractal and fractional parameters simultaneously. We observe from these numerical results that by varying the fractal and fractional orders, the dynamics of infected compartments in both humans and mosquitos decrease much faster than that when using integer order equations, either by varying only fractal order or only fractional orders. Thus, it is concluded that the fractalfractional operator provides a better understanding for the epidemic disease model with real data.

\section{Conclusions}

We obtained a dengue fever model in the framework of fractal-fractional operator. We obtained the basic reproduction number for the proposed dengue model equal to $\mathcal{R}_{0} \approx$ 2.2020 for the real cases of East Java, Indonesia, for 2018. We presented the model stability results and found that the model is locally asymptotically stable at the disease-free case when $\mathcal{R}_{0}<1$. Then, the application of fractal-fractional operator in the sense of CaputoFabrizio was applied to the model, and we obtained a generalized model. The latter model was then used to present a novel numerical procedure and solution. The numerical re- 
sults for the fractal and fractional orders have been compared with the real cases of dengue fever, and we have found that, by varying the values of the fractal and fractional orders arbitrary, the best fitting has been obtained. Moreover, we used the fractal and fractional order parameter values and presented numerous graphical results for the model. We fixed the fractal order and varied the fractional order, showing the graphical results. We also fixed the fractional order and varied the fractal order to obtain the numerical results. Similarly, we varied both fractal and fractional orders equally and unequally and obtained many graphical results. Based on these numerical results, we came to a conclusion that varying both fractal and fractional orders provides the best results for the minimization of infected compartments in mosquitoes and humans and for the increase of the noninfected compartments of humans and mosquitos by decreasing the fractal and fractional orders. From the present analysis of the dengue infection model, we suggest to the readers that the application of fractal-fractional operators to a real life problem provides better results than using the ordinary order.

Acknowledgements

All the data available without any restrictions.

Funding

This work was supported by Universitas Airlangga through Hibah Riset Mandat (No. 342/UN3.14/LT/2019).

Availability of data and materials

Please contact author for data requests.

Competing interests

The authors declare that they have no competing interests.

\section{Authors' contributions}

The main idea of this paper was proposed by F and MAK. The mathematical results are obtained by CA, F and MAK. The numerical experiment was performed by MAK. F, CA, MAK, EA revised the results and improved the paper. All authors read and approved the final manuscript.

\section{Author details}

'Department of Mathematics, Faculty of Science and Technology, Universitas Airlangga, 60115, Surabaya, Indonesia. ${ }^{2}$ Informetrics Research Group, Ton Duc Thang University, Ho Chi Minh City, Vietnam. ${ }^{3}$ Faculty of Mathematics and Statistics, Ton Duc Thang University, Ho Chi Minh City, Vietnam. ${ }^{4}$ Department of Mathematics, Faculty of Science, King Abdulaziz University, P. O. Box 80203, Jeddah 21589, Saudi Arabia.

\section{Publisher's Note}

Springer Nature remains neutral with regard to jurisdictional claims in published maps and institutional affiliations.

Received: 4 June 2020 Accepted: 4 August 2020 Published online: 14 August 2020

\section{References}

1. Centres for disease control and prevention: Dengue (2016). https://www.cdc.gov/dengue/

2. Chowell, G., Diaz-Duenas, P., Miller, J., Alcazar-Velazco, A., Hyman, J., Fenimore, P., Castillo-Chavez, C.: Estimation of the reproduction number of dengue fever from spatial epidemic data. Math. Biosci. 208(2), 571-589 (2007)

3. Shekhar, C.: Deadly dengue: new vaccines promise to tackle this escalating global menace. Chem. Biol. 14(8), $871-872$ (2007)

4. World health organization (WHO): Dengue and severe dengue

5. Nathin, M., Harun, S., Sumarmo: Dengue haemorrhagic fever and Japanese B encephalitis in Indonesia. Southeast Asian J. Trop. Med. Public Health 19(3), 475-481 (1988)

6. Ministry of the Health of the Republic of Indonesia, Dengue Hemorrhagic Fever Situation (2016). https://pusdatin kemkes.go.id/article/view/16090700001/situasi-demam-berdarah-dengue-2016.html

7. Esteva, L., Vargas, C.: Influence of vertical and mechanical transmission on the dynamics of dengue disease. Math. Biosci. 167(1), 51-64 (2000)

8. Derouich, M., Boutayeb, A.: Dengue fever: mathematical modelling and computer simulation. Appl. Math. Comput. 177(2), 528-544 (2006)

9. Rodrigues, H.S., Monteiro, M.T.T., Torres, D.F.: Dynamics of dengue epidemics when using optimal control. Math. Comput. Model. 52(9-10), 1667-1673 (2010)

10. Rodrigues, H.S., Monteiro, M.T.T., Torres, D.F.: Vaccination models and optimal control strategies to dengue. Math. Biosci. 247, 1-12 (2014) 
11. Dorigatti, I., McCormack, C., Nedjati-Gilani, G., Ferguson, N.M.: Using wolbachia for dengue control: insights from modelling. Trends Parasitol. 34(2), 102-113 (2018)

12. Agusto, F., Khan, M.: Optimal control strategies for dengue transmission in Pakistan. Math. Biosci. 305, 102-121 (2018)

13. Anggriani, N., Tasman, H., Ndii, M.Z., Supriatna, A.K., Soewono, E., Siregar, E.: The effect of reinfection with the same serotype on dengue transmission dynamics. Appl. Math. Comput. 349, 62-80 (2019)

14. Champagne, C., Paul, R., Ly, S., Duong, V., Leang, R., Cazelles, B.: Dengue modeling in rural Cambodia: statistical performance versus epidemiological relevance. Epidemics 26, 43-57 (2019)

15. Sardar, T., Rana, S., Chattopadhyay, J:: A mathematical model of dengue transmission with memory. Commun. Nonlinear Sci. Numer. Simul. 22(1-3), 511-525 (2015)

16. Qureshi, S., Atangana, A.: Mathematical analysis of dengue fever outbreak by novel fractional operators with field data. Phys. A, Stat. Mech. Appl. 526, 121127 (2019)

17. Khan, M.A., Kolebaje, O., Yildirim, A., Ullah, S., Kumam, P., Thounthong, P.: Fractional investigations of zoonotic visceral leishmaniasis disease with singular and non-singular kernel. Eur. Phys. J. Plus 134(10), 481 (2019)

18. Jan, R., Khan, M.A., Kumam, P., Thounthong, P.: Modeling the transmission of dengue infection through fractional derivatives. Chaos Solitons Fractals 127, 189-216 (2019)

19. Wang, W., Khan, M.A., Kumam, P., Thounthong, P., et al.: A comparison study of bank data in fractional calculus. Chaos Solitons Fractals 126, 369-384 (2019)

20. Khan, M., Khan, A., Elsonbaty, A., Elsadany, A.: Modeling and simulation results of a fractional dengue model. Eur. Phys. J. Plus 134(8), 379 (2019)

21. Khan, M.A.: The dynamics of a new chaotic system through the Caputo-Fabrizio and Atangana-Baleanu fractional operators. Adv. Mech. Eng. 11(7), 1687814019866540 (2019)

22. Atangana, A.: Fractional discretization: the africans tortoise walk. Chaos Solitons Fractals 130, 109399 (2020)

23. Ghanbari, B., Atangana, A.: A new application of fractional Atangana-Baleanu derivatives: designing ABC-fractional masks in image processing. Phys. A, Stat. Mech. Appl. 542, 123516 (2019)

24. Khan, M.A., Atangana, A.: Dynamics of Ebola disease in the framework of different fractional derivatives. Entropy 21(3), 303 (2019)

25. Li, Z., Liu, Z., Khan, M.A.: Fractional investigation of bank data with fractal-fractional Caputo derivative. Chaos Solitons Fractals 2019, 109528 (2019)

26. Wang, W., Khan, M.: Analysis and numerical simulation of fractional model of bank data with fractal-fractional Atangana-Baleanu derivative. J. Comput. Appl. Math. 2019, 112646 (2019)

27. Gómez-Aguilar, J.: Chaos and multiple attractors in a fractal-fractional shinrikis oscillator model. Phys. A, Stat. Mech. Appl. 539, 122918 (2020)

28. Ghanbari, B., Gómez-Aguilar, J.: Analysis of two avian influenza epidemic models involving fractal-fractional derivatives with power and Mittag-Leffler memories. Chaos, Interdiscip. J. Nonlinear Sci. 29(12), 123113 (2019)

29. Gómez-Aguilar, J.: Multiple attractors and periodicity on the Vallis model for El niño/La niña-Southern oscillation model. J. Atmos. Sol.-Terr. Phys. 197, 105172 (2020)

30. Gómez-Aguilar, J., Atangana, A.: New chaotic attractors: application of fractal-fractional differentiation and integration. Math. Methods Appl. Sci. (2020)

31. Gomez-Aguilar, J., Cordova-Fraga, T., Abdeljawad, T., Khan, A., Khan, H.: Analysis of fractal-fractional malaria transmission model. Fractals (2020)

32. Atangana, A.: Blind in a commutative world: simple illustrations with functions and chaotic attractors. Chaos Solitons Fractals 114, 347-363 (2018)

33. Atangana, A.: Fractal-fractional differentiation and integration: connecting fractal calculus and fractional calculus to predict complex system. Chaos Solitons Fractals 102, 396-406 (2017)

34. Atangana, A., Baleanu, D.: New fractional derivatives with non-local and non-singular kernel: theory and application to heat transfer model. Therm. Sci. 20, 763-769 (2016)

35. Atangana, A., Qureshi, S.: Modeling attractors of chaotic dynamical systems with fractal-fractional operators. Chaos Solitons Fractals 123, 320-337 (2019)

36. Van Den Driessche, P., Watmough, J.: Reproduction numbers and sub-threshold endemic equilibria for compartmental models of disease transmission. Math. Biosci. 180(1-2), 29-48 (2002) Cited by 2672. https://doi.org/10.1016/S0025-5564(02)00108-6

37. Ministry of Health of the Republic of Indonesia, East Java Province Health Profile 2017, Indonesia (2018)

38. Provided by Health Office (Dinas Kesehatan) of East Java Province, D.: Indonesia

39. East Java Province, C.B.o.S.s Indonesia. (2018) https://jatim.bps.go.id/dynamictable/2018/04/16/394/angka-harapanhidup-ahh-penduduk-jawa-timur-menurut-kabupaten-kota-ipm-metode-baru-2010-2017.html

40. Manore, C.A., Hickmann, K.S., Xu, S., Wearing, H.J., Hyman, J.M.: Comparing Dengue and Chikungunya emergence and endemic transmission in A. aegypti and A. albopictus. J. Theor. Biol. 356, 174-191 (2014) 